\title{
Sequentially Mixed Search and Equilibrium Price Dispersion*
}

\author{
Shouyong Shi \\ Pennsylvania State University \\ (sus67@psu.edu)
}

This version: October 2018

\begin{abstract}
Many markets feature sequentially mixed search (SMS), which has directed search in the first stage followed by noisy matching with multiple offers in the second stage. I construct a simple model of SMS, establish existence of a unique equilibrium, analyze how the two stages of SMS interact to affect quantities and price dispersion, and conduct comparative statics with respect to the meeting efficiency and the economic condition. By extending the model to endogenize search effort, I show that the equilibrium is not constrained socially efficient. Policies are introduced to restore efficiency and to manage aggregate demand and supply.
\end{abstract}

JEL classification: D40; D60; D83.

Keywords: Sequentially mixed search; Price dispersion; Efficiency.

* Address: Shouyong Shi, 502 Kern Building, Pennsylvania State University, University Park, PA 16802, USA. 


\section{Introduction}

A real estate agent often asks a house buyer what price range and neighborhood the buyer is searching for. The agent gives a list of houses on the market that fit into the buyer's description and the buyer chooses some of these houses to visit. Similarly, when searching for a job, a worker has a desired range of wages and restricts the applications to such wages. Search returns several job openings that fit into the worker's desired range and the worker chooses whether to accept any one of them. In the market for goods and services, a consumer narrows down search to a specific range of prices and conditions and, after getting a number of quotes in that range, chooses whether to trade. An example is online booking of airline tickets or hotel rooms.

The common feature of all these markets is sequentially mixed search (SMS) - directed search followed by noisy matching. In the first stage, individuals choose to restrict search by a feature of the price distribution, such as the price range or the maximum price, with the rational expectation that the rate of meeting a price offer will depend on the specified feature. This dependence is quintessential in directed search. ${ }^{1}$ For example, lowering the maximum house price to search for reduces the probability of finding such a house in any given neighborhood. In contrast to purely directed search, a searcher in this stage does not know the price or the pricing mechanism he/she will encounter. The second stage is noisy matching, as in Burdett and Judd (1983). A searcher randomly receives a number of meetings that satisfy the feature specified in the first stage. Crucially, this number can be more than one. A searcher chooses which meeting to accept. Individuals who post prices anticipate this ex post competition for the same searcher.

Given the ubiquitous nature of SMS, it is surprising that it has not received much attention in the literature. The literature formulates search either as purely directed search, which excludes noisy matching in the second stage, or as completely undirected search. ${ }^{2}$

\footnotetext{
${ }^{1}$ Well-known examples of directed search models are Peters (1991), Montgomery (1991), Moen (1997), Julien et al. (2000), Burdett et al. (2001), and Shi (2001).

${ }^{2}$ The literature on undirected search and/or price dispersion goes back at least to Diamond (1971), Shilony (1977), Salop and Stiglitz (1977), and Varian (1980), to mention a few. For directed search models, see the previous footnote and the additional ones at the end of this Introduction.
} 
In this paper, I construct a simple model of SMS to study the positive and normative implications. Focusing on the interaction between the two stages of SMS, the positive analysis will examine how directed search regulates price dispersion and search effort, and how the equilibrium responds to changes in the meeting efficiency and the value of a trade. The normative analysis will investigate when the equilibrium is socially inefficient, how policies can correct the inefficiency, and how these policies manage aggregate demand and supply efficiently.

Section 2 constructs the baseline model of SMS and establishes existence of a unique equilibrium. There is a unit measure of visitors, each wanting to consume one unit of a good, and an endogenous measure of stayers who post prices to sell the good. In the stage of directed search, individuals choose a submarket to enter, where a submarket is described by the maximum price, $p_{H}$, and the meeting rate for a visitor, $x$. This choice takes into account the equilibrium relationship between $x$ and $p_{H}$. Stayers' entry is competitive under a fixed cost. Every stayer entering submarket $\left(p_{H}, x\right)$ commits to posting prices no higher than $p_{H}$, but can post any price that does not exceed $p_{H}$. In each submarket, an aggregate meeting function with constant returns to scale determines the measure of meetings. A visitor receives meetings according to the Poisson process at the rate $x$ and, at the end of a period, chooses which meeting to accept for trade. With identical individuals on each side of the market, only one submarket is active in the equilibrium.

The sequentially mixed search equilibrium (SMSE) differs from both purely directed search and purely noisy search. The main difference from purely directed search is noisy matching in the second stage of SMS that generates multiple meetings for a visitor and a continuous price distribution. Although this is similar to purely noisy search in the Burdett-Judd (1983) model, directed search in the first stage of SMS makes the equilibrium different both positively and normatively. On the positive side, directed search in the first stage of SMS regulates the equilibrium price distribution by determining $x$. In particular, $p_{H}$ in the SMSE is strictly lower than a visitor's value of not trading. These two objects are equal to each other in the Burdett-Judd model. See section 3.1 for more detail. On the normative side, the SMSE is constrained efficient in the baseline model, in contrast 
to the inefficient equilibrium under purely noisy search. The contrast reflects the value of commitment. Although visitors have the option not to commit to $p_{H}$, they optimally choose to commit because the commitment increases the expected surplus. Competing for visitors who want to commit to $p_{H}$, stayers also choose optimally to commit.

The SMSE has interesting comparative statics, as examined and explained in section 3.3. In particular, an increase in the meeting efficiency induces not only more stayers to enter the market, but also visitors to choose a submarket that has a higher meeting rate $x$. Posted and transaction prices fall in the first-order stochastic dominance. However, price dispersion widens. This result helps explain the empirical finding that the Internet has not reduced price dispersion, e.g., Baye et al. (2004), Ellison and Ellison (2005).

Section 4 endogenizes visitors' search effort. After entering a submarket, each visitor chooses search effort that increases the visitor's meeting rate. A unique SMSE exists. Similar to an increase in the meeting efficiency, an exogenous reduction in the cost of search effort reduces prices but widens price dispersion. In contrast to the baseline model, the SMSE is not constrained efficient. When individual visitors increase search effort, they increase the return on search to other visitors, resulting in higher search effort in the equilibrium and higher congestion. This externality is not internalized by directed search, because visitors choose search effort after the stage of directed search. Relative to the constrained social optimum, the SMSE has excessive search effort, an inefficiently high meeting rate for a visitor, a deficient meeting rate per search effort for a visitor, and excessive aggregate output. As a result, the SMSE has inefficiently wide dispersion in prices, although price levels are lower than in the SMSE than in the social optimum. Price dispersion is necessary for the inefficiency, because the externality comes from visitors increasing search effort to find lower prices.

The following policies can restore social efficiency of the SMSE and maintain a balanced budget: a proportional subsidy to stayers' entry cost, a proportional tax on the joint value of a trade, and a lump-sum rebate to visitors. This is explained in section 4.2. I also examine how the corrective policies respond to an increase in the joint value of a trade. In 
the absence of the policies, an increase in the joint value of a trade increases visitors' search effort excessively in the SMSE, resulting in an overheated economy. The meeting rate for a visitor, aggregate output and price dispersion all increase by too much to be socially efficient. The corrective policies described above help cool down the economy. Moreover, these policies may need to respond to the economic conditions aggressively in the sense that the subsidy rate to stayers' entry cost and the tax on the joint value of a trade increase in response to an increase in the joint value of a trade. This policy recommendation is opposite to that by Diamond (1982), who argues for policies to stimulate aggregate demand under the assumption that the matching function has increasing returns to scale.

One contribution of this paper is to construct and analyze a tractable framework of SMS. Another contribution is to analyze how policies can help manage aggregate activities with endogenous search effort. Since directed search models have existed for some time and the Burdett-Judd model of noisy matching for even a longer time, one might wonder why the literature has not integrated the two to study SMS. One possible reason is that the BurdettJudd model treats the number of meetings for a visitor as the primary characteristic. Grafting directed search onto the Burdett-Judd model would make this number a choice of a visitor, which is an intractable integer problem. Instead, I formulate directed search as a continuous choice of the Poisson arrival rate of meetings to a visitor, $x$, together with the price bound $p_{H}$. This formulation greatly simplifies the model and enables me to conduct the analysis with a general meeting function.

Three additional contrasts to previous work are useful. First, some papers have allowed stayers to direct visitors' search with mechanisms instead of prices, e.g., auctions with reserve prices (Julien et al., 2000), potentially vague messages (Menzio, 2007), list prices with bargaining (Stacey, 2015) or with sequential inspection (Lester et al., 2017), and auctions with cheap talk (Kim and Kircher, 2015). A common assumption of these models is that a visitor cannot participate in more than one stayer's mechanism and, hence, cannot have multiple meetings that occur in SMS.

Second, SMS differs substantially from directed search with multiple applications, e.g., 
Albrecht et al. (2006) and Galenianos and Kircher (2009). In the latter models, as in models of purely directed search, each stayer can precisely direct a visitor's search with a price or a pricing mechanism. When applying to a stayer, a visitor knows exactly the price or the pricing mechanism that he/she will get if there is a meeting. In contrast, the prices a visitor encounters in SMS are random draws from the equilibrium distribution. Besides realism, noisy matching in the second stage of SMS affects the equilibrium. In Galenianos and Kircher (2009), the number of equilibrium prices is equal to the number of applications that a worker can send out simultaneously. With SMS, the support of equilibrium prices is a connected interval. Also, the equilibrium in Galenianos and Kircher (2009) is not constrained efficient, but the SMSE is constrained efficient in the baseline model here. Inefficiency of the SMSE arises from endogenous search effort, instead.

Finally, another strand of literature has studied a different type of mixed search. Lester (2011) models a market where informed buyers direct search in one submarket while uninformed buyers have undirected search in another submarket. Godoy and Moen (2013) study the co-existence of the two modes of search, and Delacroix and Shi (2013) examine the coexistence of directed search and bargaining. In these papers, mixing occurs across submarkets - There is no mixing of different modes of search in any given submarket. Moreover, these papers do not allow for multiple meetings for a visitor. ${ }^{3}$

\section{Baseline Model}

\subsection{The model environment}

Consider a one-period economy with a continuum of homogeneous individuals on each side of the market. On one side are stayers whose measure is elastically determined by competitive entry at the entry cost $k>0$. The cost of producing a good is normalized to 0 . On the other side are visitors whose measure is relatively inelastic and, for simplicity, fixed at unity. A visitor wants to consume one unit of an indivisible good and a stayer can

\footnotetext{
${ }^{3}$ As an interesting twist of Lester's (2011) model, Bethune et al. (2018) introduce noisy search into the directed search submarket. However, they take the meeting rate as exogenous instead of modeling it as part of the choice of directed search.
} 
produce one unit. Stayers post prices and visitors search. A trade generates a joint value $y$, the stayer obtains a surplus $p$, and the visitor gets $(y-p)$, where $p$ is the price. In the event of failing to trade, a stayer obtains 0 and a visitor obtains a value $b$.

Search is sequentially mixed (SMS), with directed search followed by noisy matching. In the first stage, individuals choose which submarket to enter, where stayers' entry is competitive. There is a continuum of potential submarkets indexed by $\left(p_{H}, x\right)$, where $p_{H}$ is the upper bound on prices in the submarket and $x$ is the arrival rate of a meeting to a visitor. By entering submarket $\left(p_{H}, x\right)$, stayers commit to posting prices not exceeding $p_{H}$ but can post any price that honors this commitment. As a characteristic of directed search, individuals take the equilibrium relationship between $x$ and $p_{H}$ into account and make the optimal tradeoff between the meeting rate and the match surplus. They also form rational expectations on the distribution of posted prices and the lower bound on prices in the submarket. However, visitors do not observe the price posted by a stayer before meeting the stayer. The second stage is noisy matching in each submarket. Given the measures of stayers and visitors in a submarket, a meeting function, described below, determines the measure of meetings in a period and delivers the meeting rate for a visitor, $x$. At this rate, a visitor receives meetings according to the Poisson process. At the end of the period, a visitor chooses which of the received meetings to accept. An accepted meeting is called a match, which yields immediate trade at the posted price. For simplicity, I assume that a stayer receives at most one meeting in the period. ${ }^{4}$

In submarket $\left(p_{H}, x\right)$, let $N_{s}$ be the measure of stayers and $N_{v}$ the measure of visitors. The meeting function is $M\left(N_{s}, N_{v}\right)$, which has constant returns to scale. For a visitor, the probability of receiving a number $j$ of meetings in the period is $\frac{x^{j} e^{-x}}{j !}$, and so the expected number of meetings is $x$. Since the measure of meetings is equal to both $x N_{v}$ and $M\left(N_{s}, N_{v}\right)$, equating the two yields $x$ that is consistent with the entry decision:

$$
x=\frac{M\left(N_{s}, N_{v}\right)}{N_{v}}=M(\theta, 1),
$$

where $\theta \equiv \frac{N_{s}}{N_{v}}$ is the tightness of the submarket. For any given $x$, define $\theta(x)$ as the

\footnotetext{
${ }^{4}$ See Shi (2018) for the version of the model with many-to-many meetings where every individual, regardless of the side of the market, can have more than one meeting in a period.
} 
solution for $\theta$ to (2.1). This is the tightness needed to deliver the meeting rate $x$ for each visitor in the submarket. A stayer receives a meeting in the period with the probability $\lambda(x) \equiv \frac{M\left(N_{s}, N_{v}\right)}{N_{s}}=\frac{x}{\theta(x)}$.

A critical feature of noisy matching is that a visitor can receive multiple meetings before choosing one to trade. As in the Burdett-Judd model, the underlying assumption is that a visitor can choose a trade only after receiving all meetings in a period, rather than make the choice whenever a meeting arrives. This assumption may reflect a fixed cost of making decisions on offers, or simply the inability to decide on an offer on spot. With this feature, a stayer in a meeting faces potential competitors for the same visitor. As noted in the Introduction, the modeling of noisy matching here is substantially simpler than in the Burdett-Judd model, because I use the continuous variable $x$ as the primary characteristic of the meeting process, instead of the integer number of meetings. Also, the meeting function is general in my model.

Many markets have the features of SMS. In the labor market, firms (vacancies) are stayers and job applicants are visitors. In the goods market, sellers are stayers and buyers are visitors. In the asset market, sellers can be stayers in some segments of the market but visitors in other segments. ${ }^{5}$ To help understand SMS, suppose that the market is the labor market. Define $p=y-w$, where $w$ is the wage. Then, submarkets are indexed by the meeting rate and the lowest wage $w_{L}$, i.e., a worker's reservation wage. A submarket with a higher reservation wage is likely to have a smaller number of vacancies and, hence, a lower meeting rate of an offer to each applicant. Taking this relationship into account, each worker chooses the submarket to participate. Once in the submarket, a worker applies to a large number of vacancies randomly. After receiving applications, a firm can choose one, and only one, of the applicants to send an offer. The offer reaches an applicant in time (in the period) with the probability $\lambda(x)$. The aggregate measure of contacts (offers)

\footnotetext{
${ }^{5}$ In a directed search environment, Shi and Delacroix (2018) study the issue of which side should incur the cost to organize trade in order to maximize social welfare in an economy with search frictions. In the current model, the entry cost $k$ is the elastic side's participation cost in the market, while the participation cost of the inelastic side and the cost of creating a trading post is zero. Under these assumptions, the analysis in Shi and Delacroix (2018) implies that social welfare is the same regardless of whether the elastic side or the inelastic side posts prices to organize trade.
} 
is the value of the meeting function. A worker can receive multiple offers and choose one to accept. The aggregate measure of acceptance is the measure of matches.

Let me return to the general environment of the model. Denote $F(p \mid x)$ as the cumulative distribution function of posted prices in submarket $\left(p_{H}, x\right)$. The upper bound on prices is $p_{H}$ and the lower bound is $p_{L}$. Consider a stayer $S$ in submarket $\left(p_{H}, x\right)$ and let $p$ be the price posted by $S$. To compute the trading probabilities, I use a property of the Poisson distribution in the following lemma:

Lemma 2.1. If $Z_{1}$ is Poisson with the rate $\zeta$ and $Z_{2} \mid\left(Z_{1}=j\right)$ is binomial with $(j, q)$, then $Z_{2}$ is Poisson with the rate $\zeta q$ (see Haight, 1967, p.46). This implies that, conditional on having a meeting, the trading probability for stayer $S$ is $e^{-x F(p \mid x)}$. For a visitor, the probability of trading at a price no higher than $p$ is $\left[1-e^{-x F(p \mid x)}\right]$.

To illustrate the above property of the Poisson distribution and its implications, suppose that stayer $S$ met a visitor $V$. Let $n$ be the number of visitor $V$ 's meetings, other than the one with stayer $S$, that have prices no higher than $p$. Conditional on the meeting, the trading probability of stayer $S$ is $\operatorname{Pr}(n=0){ }^{6}$ Because meetings arrive to $V$ independently from different sellers, the number of $V$ 's meetings other than the one with $S$ is still Poisson distributed with the rate $x$. That is, this number is equal to $j$ with the probability $\frac{x^{j} e^{-x}}{j !}$. In each of those meetings, the price is not higher than $p$ with the probability $F(p \mid x)$. Thus, $n$ conditional on $j$ is binomial with $(j, F(p \mid x))$. The property of the Poisson distribution described in Lemma 2.1 implies that $n$ unconditional on $j$ is Poisson with the rate $x F(p \mid x)$. Hence, the probability of $n=0$ is $e^{-x F(p \mid x)}$. To verify this result, note that if $V$ has $j$ other meetings, $V$ trades with $S$ when all those meetings have prices higher than $p$. This event occurs with the probability $\operatorname{Pr}(n=0 \mid j)=[1-F(p \mid x)]^{j}$. Unconditional on $j$, stayer $S$ expects visitor $V$ to accept the match with the following probability:

$$
\operatorname{Pr}(n=0)=\sum_{j=0}^{\infty} \frac{x^{j} e^{-x}}{j !}[1-F(p \mid x)]^{j}=e^{-x F(p \mid x)} .
$$

\footnotetext{
${ }^{6}$ If a visitor receives two or more identical prices that are the lowest among the received offers, the visitor chooses one of them with equal probability. It will become clear later that the offer distribution contains no mass point. Thus, the probability that stayer $S$ faces a competitor who offers the same price and has met the same visitor is zero.
} 
This verifies the result for stayer $S$ stated in Lemma 2.1. Similarly, for a visitor, let $n$ be the number of meetings with prices no higher than $p$. Conditional on the number of meetings, $j$, the number $n$ is binomial with $(j, F(p \mid x))$. Unconditional on $j$, the number $n$ is Poisson with the rate $x F(p \mid x)$. Hence, the probability for the visitor to trade at a price no higher than $p$ is $\operatorname{Pr}(n \geq 1)=1-e^{-x F(p \mid x)}$, as stated in Lemma 2.1.

Conditional on having a meeting, a stayer's expected surplus is

$$
\Pi(p, x, F) \equiv p e^{-x F(p \mid x)} .
$$

Denote the maximum of a stayer's expected surplus in a meeting as

$$
\pi(x)=\max _{p \in \operatorname{supp}(F)} \Pi(p, x, F) .
$$

A stayer's expected profit of entering submarket $\left(p_{H}, x\right)$ is $\lambda(x) \pi(x)-k$, where $\lambda$ is the stayer's meeting probability in the period and $k$ the entry cost. For a visitor, the expected surplus to a visitor in the submarket is:

$$
D(x) \equiv \int_{p_{L}}^{p_{H}}(y-p-b) \mathrm{d}\left[1-e^{-x F(p \mid x)}\right],
$$

where the derivative $d$ is with respect to $p$.

The trading process endogenously generates an aggregate matching function and a distribution of transaction prices. To see this, note that a visitor trades with the probability $1-e^{-x}$. The measure of matches in the period is $N_{v}\left(1-e^{-x}\right)$. This matching function has constant returns to scale. Conditional on having at least one meeting, a visitor succeeds in trading at a price no higher than $p$ with the probability:

$$
G(p \mid x) \equiv \frac{1-e^{-x F(p \mid x)}}{1-e^{-x}} .
$$

This is the cumulative distribution of transaction prices. It is clear that $G(p \mid x) \geq F(p \mid x)$ for all $p$, with a strict inequality for all $p \in\left(p_{L}, p_{H}\right)$. That is, transaction prices are lower than posted prices in the first-order stochastic dominance. Thus, search enables visitors to obtain lower prices than posted prices. 
A sequentially mixed search equilibrium (SMSE) consists of a tightness function, $\theta(x)$, stayers' choices of the submarket to enter and the price to post, visitors' choices of the submarket to enter, a collection of active submarkets, $\left(p_{H i}, x_{i}\right)_{i \in I}$ for some set $I$, and the implied distribution of posted prices, $\left(F_{i}\right)_{i \in I}$, that satisfy (i)-(iii) below:

(i) For each $i \in I$, a stayer in submarket $i$ optimally chooses the price $p$ to post to maximize $\Pi(p, x, F)$ under the constraint $p \leq p_{H i}$. The maximized $\Pi$ is $\pi(x)$, and posted prices across stayers generate the distribution $F_{i}$.

(ii) Given $\theta($.$) , a visitor chooses the submarket to enter to maximize D(x)$ subject to the dependence of $p_{H}$ and $F$ on $x$. Each submarket $i \in I$ is entered by some visitors.

(iii) The tightness function $\theta(x)$ satisfies a complementary slackness condition on competitive entry of stayers: $\lambda(x) \pi(x)=k$ for all submarkets with $\pi(x) \geq k$ and $\theta(x)=0$ for all submarkets with $\pi(x)<k$.

This definition captures directed search by the choice of $x$ in (ii), which incorporates how $p_{H}$ and the distribution $F$ depend on $x$. Noisy matching allows for the possibility that a visitor has multiple meetings, which is built into the expressions for $D$ and $\Pi$. Requirements (i) and (ii) are about active submarkets in the SMSE. In contrast, requirement (iii) is about all submarkets, where the tightness appears through $\lambda(x)$. This requirement restricts the beliefs on the function $\theta(x)$ in inactive submarkets as well as in active submarkets, which is commonly imposed in directed search models (e.g., Menzio and Shi, 2010, and Guerrieri, et al., 2010). For every active submarket, the expected profit of a stayer should be zero. For every inactive submarket, a stayer should be unwilling to enter the submarket even if the stayer would meet a visitor with probability one in the submarket. This requirement prevents a submarket from being inactive just because individuals on each side expect no one on the other side will enter the submarket.

\subsection{Optimal choices and the SMSE}

To characterize this optimal decision, define $\bar{x} \leq \infty$ by $\theta(\bar{x})=\infty$ as the natural upper bound on $x$, and define $\varepsilon(x) \equiv \frac{x \theta^{\prime}(x)}{\theta(x)}$. 
Assumption 1. The meeting function is such that $\theta^{\prime}(x)>0$ and $\theta^{\prime \prime}(x)>0$ for all $x \in(0, \bar{x})$, and that $\theta(0)=0, \theta^{\prime}(0)>0$, and $\theta^{\prime}(\bar{x})=\infty$. Also, $\varepsilon(x)>1$ for all $x>0$, and $\theta^{\prime}(0) k<y-b$.

The assumptions on $\theta$ are equivalent to the following standard properties of the meeting function: $M$ is strictly increasing and concave in each of the two arguments, $M(0,1)=0$, $M_{1}^{\prime}(0,1)<\infty$ and $M_{1}^{\prime}(\infty, 1)=0 .{ }^{7}$ These assumptions imply $\lambda^{\prime}(x)<0$ and $\lambda(\bar{x})=0$. Because $\varepsilon=1-\theta \lambda^{\prime}$, the feature $\varepsilon(x)>1$ is implied by $\lambda^{\prime}(x)<0$, but it is listed above for a reference. The assumption $\theta^{\prime}(0) k<y-b$ requires the joint surplus of a trade to exceed the cost of stayers' entry that is needed to increase a visitor's meeting rate marginally above zero. If this assumption is violated, it is socially efficient to shut down the market.

To analyze optimal choices, consider a stayer first. The individual makes two decisions sequentially: which submarket to enter and what price to post in the submarket. Suppose that the stayer is already in submarket $\left(p_{H}, x\right)$. The stayer chooses the posted price $p \leq p_{H}$ to maximize the expected surplus in a meeting, $\Pi(p, x, F)$, as in $(2.2)$. The maximized expected surplus is $\pi(x)$. If a price $p \leq p_{H}$ is not in the support of $F$, then it must be the case that $\Pi(p, x, F)<\pi(x)$. Conversely, all prices in the support of $F$ must yield the same expected payoff to a stayer in a meeting as $\pi(x)$. That is,

$$
\Pi(p, x, F)=\pi(x) \text { for all } p \in \operatorname{supp}(F) .
$$

This equal-surplus condition determines the distribution of offers in the submarket:

$$
F(p \mid x)=\frac{1}{x} \ln \left(\frac{p}{\pi(x)}\right) \text { for all } p \in \operatorname{supp}(F) .
$$

It is convenient to invert $F$ as

$$
p=\pi(x) e^{x F} \text { for all } p \in \operatorname{supp}(F)
$$

Because the bounds on the support must satisfy $F\left(p_{L}\right)=0$ and $F\left(p_{H}\right)=1$, then ${ }^{8}$

$$
p_{L}=\pi, \quad p_{H}=\pi(x) e^{x} .
$$

\footnotetext{
${ }^{7}$ In particular, because $M\left(N_{s}, N_{v}\right)=N_{v} M(\theta, 1)$, then $M_{2}^{\prime}\left(N_{s}, N_{v}\right)=x-\theta M_{1}^{\prime}(\theta, 1)$. The property $M_{2}^{\prime}\left(N_{s}, N_{v}\right)>0$ and the fact $M_{1}^{\prime}(\theta, 1)=1 / \theta^{\prime}(x)$ imply $\varepsilon(x)>1$.

${ }^{8}$ The price $p_{H}$ should satisfy a visitor's individual rationality: $p_{H} \leq y-b$. I will show later in Corollary 3.1 that this inequality holds strictly in the equilibrium, and so the constraint is not binding.
} 
The stayer's expected profit of entering submarket $\left(p_{H}, x\right)$ is $\lambda(x) \pi(x)-k$. In every submarket with $\pi(x)<k$, the tightness is $\theta(x)=0$. In every submarket with $\pi(x) \geq k$, a stayer's expected profit of entry is zero. This yields:

$$
\pi(x)=\frac{k}{\lambda(x)}
$$

To fit (2.6)-(2.9) into the equilibrium definition, it is useful to read them in the following order. The formula for $p_{H}$ in (2.8) is the relationship between $x$ and $p_{H}$ needed for directing entry decisions. Competitive entry by stayers determines the function $\pi(x)$, as in (2.9). Given the dependence of $x$ on $p_{H}$ and the function $\pi(x)$, stayers optimally choose the price to post under the constraint $p \leq p_{H}$. This choice yields the distribution $F$, as in (2.6), and the lower bound on the support, $p_{L}$, as in (2.8). Notice that $\pi(x)$ given by (2.9) is a strictly increasing function under Assumption 1. In order to deliver a higher meeting rate to a visitor, there must be a larger measure of stayers who enter the submarket. As a result, the meeting probability for a stayer is low. To induce the increased entry in this case, the expected surplus in a meeting must increase for a stayer.

Now consider a visitor. If the visitor participates in submarket $\left(p_{H}, x\right)$, the expected surplus is $D(x)$, as given by (2.4). The visitor chooses $x$ to maximize $D(x)$, taking the relationships (2.6) and (2.9) into account. Substituting $p$ from (2.7) into (2.4) to compute the integral and then substituting $\pi$ from (2.9), I have:

$$
D(x)=(y-b)\left(1-e^{-x}\right)-k \theta(x)
$$

For future references, it is useful to interpret the last term in (2.10), which is equal to $\pi(x) x$ instead of $\pi(x)$. This is the expected surplus that the visitor concedes to a stayer in the submarket. The appearance of the product between $\pi$ and $x$ should not be construed as suggesting that a visitor can have more than one trade. Rather, a visitor has at most one trade in the period. However, a stayer "prices in" the risk of losing a visitor to a competitor. The potential failure to trade with a visitor in a meeting raises the price that the stayer charges in order to break even for entry, resulting in a reduction in a visitor's expected surplus by $\pi(x) x$. 
Because $\theta(x)$ is a strictly convex function, $D(x)$ is strictly concave and, hence, is maximized by a unique $x$. Denote the unique maximizer as $x^{*}$. If $x^{*}$ is interior, then it satisfies the first-order condition:

$$
\theta^{\prime}\left(x^{*}\right) e^{x^{*}}=\frac{1}{k}(y-b) .
$$

It is easy to verify $x^{*}<\bar{x}$, where $\theta(\bar{x})=\infty$. Also, $x^{*}>0$ under the maintained assumption $y-b>\theta^{\prime}(0) k$. The above analysis has established the following proposition:

Proposition 2.2. A unique SMSE exists, with only submarket $\left(p_{H}, x^{*}\right)$ being active, where $x^{*} \in(0, \bar{x})$ solves (2.11). Posted prices are continuously distributed according to $F^{*}$ over $\left[p_{L}, p_{H}\right]$, where $p_{L}$ and $p_{H}$ are given by (2.8) and $F^{*}$ by (2.6) with $x=x^{*}$. Transaction prices are distributed according to $G$ in (2.5).

\section{Equilibrium Properties, Efficiency and Interpretations}

\subsection{Directed search regulates the price distribution}

The current model encompasses two classes of search models as special cases. Purely directed search is the special case where each visitor is restricted to having at most one meeting, e.g., Peters (1991), Burdett et al. (2001) and Julien et al. (2000). Purely noisy search is the special case where $x$ is exogenous and there is no commitment to $p_{H}$, e.g., the Burdett-Judd model. Because noisy matching is the cause for price dispersion here, I focus on the link and the contrast to the Burdett-Judd model. ${ }^{9}$

To begin, it is useful to explain why noisy matching induces prices to be distributed continuously in an interval, as in the Burdett-Judd model. With noisy matching, a stayer may lose the visitor to a competitor. To increase the trading probability, a stayer would want to post a price slightly below the competitor's if he/she knew what price a competitor posts. This implies that the price distribution must be continuous. There cannot be a mass

\footnotetext{
${ }^{9}$ Burdett and Judd (1983) model noisy matching slightly differently and do not impose the unit upper bound on a stayer's capacity. These additional differences are not essential for the price distribution to be non-degenerate.
} 
point anywhere above the lowest price $p_{L}$ in (2.8): If there were, posting a slightly lower price would increase a visitor's acceptance probability by a discrete amount and, hence, increase the stayer's expected surplus. There cannot be a mass point at the lowest price $p_{L}$, either: If there were, then $p_{L}=0$, because any $p_{L}>0$ would make it profitable for a stayer to undercut the price. But if $p_{L}=0$, then posting a price $p>p_{L}$ yields a higher (positive) expected profit than posting $p_{L}$. In the equilibrium, prices are continuously distributed and stayers are mixed over prices. In addition, the support of the distribution must be a connected interval. If there were a "hole" in the support, posting a price in the hole would increase the ex post gain without reducing the acceptance probability, relative to posting the price at the lower boundary of the hole.

The main difference of the current model from the Burdett-Judd model is that the choice of a submarket is directed. To explain this difference, it is useful to translate the Burdett-Judd model into the notation of the current model. In the main analysis, Burdett and Judd (1983) fix $x$ and consider a market with a fixed measure of individuals on each side of the market. There is no commitment to any feature of the price distribution. Instead, stayers can choose any price after entering the market. Specifically, the highest equilibrium price is equal to the monopoly price; that is, $p_{H}=y-b$. The reason is that a visitor who has only one meeting is willing to accept any price that yields a positive surplus, even when this surplus is arbitrarily close to zero. Anticipating this possibility of meeting such a visitor, some stayers set the price arbitrarily close to $y-b$. Because both $x$ and $p_{H}$ are exogenous, the equation for $p_{H}$ in (2.8) determines a stayer's expected surplus in a meeting, $\pi$, that is consistent with the fixed measure of stayers.

In an extension, Burdett and Judd (1983) endogenize visitors' choice of $x$ by adding a cost of $x$, but such a choice does not direct search. Another way to endogenize $x$ in their model, which they did not use, is to allow stayers to enter the market competitively. In both extensions, there is one side of the market that does not choose which submarket to enter and, hence, participation on this side is not directed by any relationship between $x$ and the price distribution. If the Burdett-Judd model were extended to allow both for visitors to choose $x$ and for stayers to enter competitively, an equilibrium would fail to 
exist generically, because the two sides' optimal choices do not agree. Directed search in the first stage of SMS resolves this difficulty. The commitment to $p_{H}$ allows $p_{H}$ to vary with $x$ and, thereby, provides a tradeoff between the meeting rate and the match surplus.

Directed search in the first stage of SMS puts disciplines on the price distribution and the entry of stayers that are absent in the Burdett-Judd model. One discipline is $x^{*} \in(0, \infty)$ (see Proposition 2.2). Thus, the endogenous probability that a visitor receives two or more meetings, $1-(1+x) e^{-x}$, is strictly bounded in $(0,1)$. As explained above, this result is critical for the equilibrium price distribution to be non-degenerate. Visitors are able to choose to enter a submarket with no price dispersion, but this choice is inconsistent with an equilibrium. In submarket with $x=\infty$, prices are concentrated at the monopsony price, 0 . This choice is inconsistent with the equilibrium because there would be a loss to a stayer for entering such a submarket. In submarket with $x=0$, prices are concentrated at the monopoly price, $y-b$. Since the choice $x=0$ yields zero expected surplus to a visitor, visitors will not choose to enter the submarket.

More generally, the meeting rate $x$ affects the price distribution and its support. Denote the price spread as $\Delta p \equiv p_{H}-p_{L}$. The coefficient of variation in posted prices is $c v_{F} \equiv$ $\frac{\left[v_{F}\right]^{1 / 2}}{F}$, where $\mathbb{E}_{F}$ is the mean and $\operatorname{var}_{F}$ the variance of posted prices. The coefficient of variation in transaction prices is $c v_{G}$, where the distribution $G$ is used instead of $F$. These coefficients can be computed as functions of $x$ :

$$
c v_{F}(x)=\left[\frac{x\left(e^{x}+1\right)}{2\left(e^{x}-1\right)}-1\right]^{\frac{1}{2}}, \quad c v_{G}(x)=\left[e^{x}\left(\frac{1-e^{-x}}{x}\right)^{2}-1\right]^{1 / 2}
$$

The following corollary is proven in the Supplementary Appendix C: ${ }^{10}$

Corollary 3.1. An increase in $x$ increases posted and transaction prices in the first-order stochastic dominance in $F$ and $G$. Also, an increase in $x$ increases the bounds $\left(p_{L}, p_{H}\right)$, the price spread $\Delta p$, and the coefficients of variation $\left(c v_{F}, c v_{G}\right)$. In the SMSE, $p_{H}<y-b$, and so a visitor's surplus in a trade is strictly positive.

\footnotetext{
${ }^{10}$ It should be cautioned that the effects of $x$ on the price distribution stated in the corollary are partial effects, since they do not take into account changes in the parameters that cause $x$ to change. Nevertheless, the effects of $x$ are instructive. Moreover, $c v_{F}$ and $c v_{G}$ depend only on $x$. All effects of parameters on $c v_{F}$ and $c v_{G}$ must entirely go through $x$.
} 
An increase in $x$ affects the offer distribution directly by itself and indirectly through $\pi(x)$ (see (2.6)). Both effects increase prices in the first-order stochastic dominance. First, for any given $\pi$, an increase in $x$ reduces $F(p \mid x)$ for all $p$ and, hence, increases posted prices in the first-order stochastic dominance. To explain this effect, note that a higher $x$ implies that a stayer has a lower matching probability conditional on having a meeting. In this case, prices must be higher in order to generate a given expected surplus to a stayer in a meeting. Second, a higher $x$ implies a lower meeting probability for a stayer. To induce stayers to enter the submarket, the expected surplus to a stayer conditional on having a meeting, $\pi(x)$, must be higher to offset the lower meeting rate. In turn, this requires prices to be higher. Similarly, these two effects of $x$ increase transaction prices.

Of the two effects of $x$ on price distributions, the one through stayers' meeting rate is uniform across all posted prices, because meetings in a submarket are random. In contrast, the effect through a stayer's matching probability is non-uniform, because a visitor chooses which meeting to accept for trade. Conditional on having a meeting, a stayer is less likely to succeed in a trade if the posted price is higher. As a result, an increase in $x$ reduces a stayer's trading probability by more if the stayer posts a higher price. To yield the same expected surplus conditional on having a meeting, $\pi$, the price must increase by more if the price is initially higher. This is why an increase in $x$ increases the bounds on prices, the price spread and the coefficient of variation in prices.

The positive relationship between posted prices and $x$ is essential for directed search. A submarket with a higher meeting rate for a visitor necessarily has higher prices. The optimal tradeoff between the meeting rate and prices yields the equilibrium $x$. Moreover, the commitment to $p_{H}$ is important for directed search. If there were no commitment, some stayers would post the price $(y-b)$ and a visitor who happens to only meet such a stayer would accept the trade, as in the Burdett-Judd model. ${ }^{11}$ In this case, visitors' optimal choice of $x$ and stayers' competitive entry cannot both be achieved, as said above. With the commitment to $p_{H}$, a stayer can still choose to enter a submarket with $p_{H} \geq y-b$. However, this entry decision is not optimal for a stayer. The corresponding $x$ would satisfy

\footnotetext{
${ }^{11}$ Precisely, $\Pi_{1}^{\prime}\left(p_{H}^{+}, x, F\right)>0$ if $p_{H}<y-b$, where $p_{H}^{+}$is arbitrarily close to $p_{H}$ on the right-hand side.
} 
$x<x^{*}$, which would be too low to attract visitors to enter the submarket. ${ }^{12}$

\subsection{Social efficiency of the SMSE}

Another main difference of SMSE from an equilibrium with purely noisy search is constrained social efficiency. To analyze efficiency, consider a social planner who maximizes social welfare measured by the sum of expected surpluses in the economy. The planner chooses the measure of stayers to enter the market, $N_{s}$, but is constrained by the same frictions as in the market. Specifically, the planner takes as given the meeting function $M\left(N_{s}, N_{v}\right)$. If a visitor receives multiple meetings, the planner randomly chooses one of them for the visitor to trade. Total cost of stayers' entry is $k N_{s}$, and the measure of trades is $N_{v}\left(1-e^{-x}\right)$. Since a trade generates the joint surplus $(y-b)$, social welfare is:

$$
(y-b) N_{v}\left(1-e^{-x}\right)-k N_{s}
$$

To maximize social welfare, the planner chooses $N_{s}$ subject to $x=\frac{1}{N_{v}} M\left(N_{s}, N_{v}\right)$. Because $N_{v}$ is fixed, the planner's choice of $N_{s}$ is equivalent to the choice of $x$. Dividing social welfare by $N_{v}$, normalized welfare (per visitor) is:

$$
(y-b)\left(1-e^{-x}\right)-k \theta(x)
$$

where $\theta=\frac{N_{s}}{N_{v}}$ is used. This welfare measure is the same as $D(x)$ in $(2.10)$ - a visitor's expected surplus of entering a submarket $\left(p_{H}, x\right)$. Thus, the planner's choice of $x$ is identical to a visitor's choice in the SMSE. This proves the following proposition:

Proposition 3.2. The SMSE is constrained efficient.

Competitive entry of stayers and directed search are important for why the SMSE is socially efficient. With competitive entry, a stayer's profit of entering the market is zero, and so the sum of expected surpluses in the economy is equal to the sum of expected surpluses of visitors. With directed search, individuals make the tradeoff between the price

\footnotetext{
${ }^{12}$ In the context of the labor market, the result $p_{H}<y-b$ implies that an unemployed worker's reservation wage is strictly higher than the continuation value of staying unemployed, as in a typical model of directed search but different from a random search model.
} 
and the trading probability. As in most models of directed search, this tradeoff enables the equilibrium to internalize the externalities in the matching process. In contrast, the purely noisy search equilibrium, as in the Burdett-Judd model, is socially inefficient if $x$ is determined by competitive entry of stayers. In that case, $p_{H}=y-b$, which is inefficiently higher and induces excessive entry of stayers.

The literature has shown that directed search can induce constrained efficiency, even when there is two-sided heterogeneity (e.g., Shi, 2001). Against this background, I clarify that directed search does not always generate constrained efficiency. First, social efficiency of the SMSE also relies on non-rivalry among visitors in the matching stage. By assumption, a stayer meets no more than one visitor. If a visitor chooses to trade with a stayer, the choice does not reduce any other visitor's chance of trade. Second, in section 4, I will show that endogenizing visitors' search effort can make the SMSE socially inefficient.

With Proposition 3.2, I can relate a visitor's optimality condition, (2.11), to the social planner's choice. When choosing the meeting rate for a visitor, the planner equates the social marginal benefit of a higher $x$ to the social marginal cost. The social marginal benefit of a higher $x$ is that it reduces the visitor's probability of failing to trade. Since the failure probability is $e^{-x}$, a marginally higher $x$ reduces this probability by $e^{-x}$. The expected social gain is $e^{-x}(y-b) N_{v}$. The marginal cost of increasing $x$ is that more stayers need to enter the market to deliver the higher $x$. To increase $x$ marginally, $\theta$ must increase by $\theta^{\prime}(x)$, and so the measure of stayers must increase by $\theta^{\prime}(x) N_{v}$. Thus, the entry cost increases in an amount $k \theta^{\prime}(x) N_{v}$. The equilibrium condition (2.11) equates this social marginal cost to the social marginal benefit of a higher $x$.

\subsection{Comparative statics}

I focus on an increase in the joint value of a trade, $y$, and in the meeting efficiency. ${ }^{13}$ For the analysis, let me modify the meeting function as $A M\left(N_{s}, N_{v}\right)$, where $A$ is the meeting efficiency. Temporarily denote $\nu=\frac{x}{A}$. The definition of $x$ implies $\nu=M(\theta, 1)$.

\footnotetext{
${ }^{13}$ One can also analyze the effects of $k$ and search cost. The effect of an increase in the entry cost, $k$, is similar to the effect of a decrease in the matching efficiency. Search cost will be analyzed in section 4.1.
} 
The solution for $\theta$ to this equation is $\theta(\nu)$ and the elasticity of this tightness function is $\varepsilon(\nu)=\frac{\nu \theta^{\prime}(\nu)}{\theta(\nu)}$. As a result, the meeting probability for a stayer is $\lambda=\frac{x}{\theta(\nu)}$. Except the change in the argument of $\theta$ and $\varepsilon$ from $x$ to $\nu$, the SMSE is characterized in the same way as before. The first-order condition for the optimal choice $x^{*}$ changes from (2.11) to

$$
\frac{1}{A} \theta^{\prime}\left(\frac{x^{*}}{A}\right)=\frac{y-b}{k} e^{-x^{*}} .
$$

The following proposition is proven in the Supplementary Appendix C:

Proposition 3.3. (i) $\frac{d x^{*}}{d y}>0, \frac{d \theta}{d y}>0, \frac{d p_{L}}{d y}>0, \frac{d p_{H}}{d y}>0, \frac{d \Delta p}{d y}>0$, and prices increase in $y$ in the first-order stochastic dominance in $F$ and $G$. (ii) $\frac{d x^{*}}{d A}>0$ and $\frac{d \theta}{d A}>0$. (iii) If $\varepsilon^{\prime} \geq 0$, then $\frac{d p_{L}}{d A}<0, \frac{d p_{H}}{d A} \leq 0$, and $\frac{d \Delta p}{d A}>0$. (iv) If $\varepsilon^{\prime} \geq 0$, an increase in $A$ reduces posted prices and transaction prices in the first-order stochastic dominance. (v) $\frac{d}{d y} c v_{i}\left(x^{*}\right)>0$ and $\frac{d}{d A} c v_{i}\left(x^{*}\right)>0$ for $i=F, G$.

An increase in $y$ increases the market tightness, increases the meeting rate for a visitor, increases the two bounds on price and shifts up the price distribution. These effects are intuitive. When the joint value of a trade increases, more stayers enter the market, which increases the market tightness. Equilibrium prices increase to be consistent with the optimal choices of both sides of the market. For visitors, the benefit of a higher $y$ induce them to enter a submarket that has a higher meeting rate, which necessarily comes with higher prices. For stayers, the meeting probability falls because of the higher entry of stayers. However, the expected surplus for a stayer in the market (unconditional on a meeting) must remain the same as the fixed cost of entry. This implies that a stayer's expected surplus conditional on a meeting must rise. For this to happen despite the decrease in the stayer's trading probability, prices must increase to raise the stayer's surplus conditional on a trade. Moreover, the increase in $x$ induced by a higher $y$ reduces the trading probability for a stayer posting a higher price by more than for a stayer posting a lower price. As explained for Corollary 3.1, this widens price dispersion.

It is important to emphasize that $y$ affects the price distribution entirely through $x$ and, hence, through directed search. If search is undirected, then $x$ is fixed, as in the 
Burdett-Judd model. In this case, competitive entry of stayers keeps $\pi(x)$ fixed, as shown by (2.9). Stayers' indifference among posted prices keeps the distribution $F$ and its support unaffected by $y$, as shown by (2.7)-(2.8). Thus, if $x$ is fixed, an increase in $y$ simply increases visitors' surplus without changing the price distribution.

Now I explain the effects of the meeting efficiency. It is then not surprising that an increase in $A$ increases the meeting rate for a visitor, $x$. However, it is ambiguous whether a higher $A$ leads to a higher meeting probability for a stayer or higher prices. The meeting probability for a stayer is $\frac{x}{\theta(\nu)}$, where $\nu=\frac{x}{A}$. Although an increases in $A$ directly increases $\frac{x}{\theta(x / A)}$, it also reduces $\frac{x}{\theta(x / A)}$ indirectly by increasing $x$. If the direct effect dominates, then a stayer's meeting probability increases in the SMSE. In this case, prices must fall in order to keep a stayer's profit of entry to be zero. Conversely, if an increase in $A$ reduces a stayer's meeting probability because the indirect effect through $x$ dominates, then prices must increase in order to keep a stayer's profit of entry to be zero.

The sensitivity of the elasticity of the tightness function, $\varepsilon(\nu)=\frac{\nu \theta^{\prime}(\nu)}{\theta(\nu)}$, determines whether a stayer's meeting probability increases in $A$. To explain, note that the marginal cost for a visitor to choose a submarket with a higher $x$ is proportional to $\frac{\theta^{\prime}(\nu)}{A}$, because stayers must be compensated for the entry in order to deliver the higher $x$. As (3.2) shows, a visitor's optimal $x$ equates this marginal cost to the expected surplus that the visitor will obtain in a trade. The quantity $\varepsilon^{\prime}$ determines how sensitive $\theta^{\prime}$ is to changes in $x$. If $\varepsilon^{\prime}$ is high, then $\theta^{\prime}$ is sensitive to changes in $x$, and the marginal cost of a higher $x$ increases sharply in $x$. In this case, it is optimal for a visitor to increase $x$ only moderately in response to a higher $A$. As a result, a stayer's meeting probability increases and prices fall. Conversely, if $\varepsilon^{\prime}$ is small, then $\theta^{\prime}$ is not sensitive to changes in $x$, and it is optimal for a visitor to increase $x$ by a large amount in response to a higher $A$. In this case, a stayer's meeting probability falls and prices rise. A sufficient condition for a higher $A$ to reduce prices is $\varepsilon^{\prime} \geq 0$, as stated in Proposition 3.3.

The condition $\varepsilon^{\prime}(\nu)>0$ is satisfied by two well-known meeting functions:

Example 3.4. The Dagum (1975) function is $\frac{M(\theta, 1)}{A}=\left(\theta^{-\rho}+1\right)^{-1 / \rho}$ with $A, \rho \in(0, \infty)$. 
This function yields $\theta(\nu)=\left[\nu^{-\rho}-1\right]^{-1 / \rho}$ and $\varepsilon(\nu)=\left[1-\nu^{\rho}\right]^{-1}$. The urn-ball matching is $\frac{M(\theta, 1)}{A}=\theta\left(1-e^{-1 / \theta}\right)$, with $A \in(0, \infty)$. This function yields $\varepsilon(\nu)=\frac{e^{1 / \theta(\nu)}-1}{e^{1 / \theta(\nu)}-1-\frac{1}{\theta(\nu)}}$. With both functions, $\lambda(0)=\bar{x}=A, \theta^{\prime}(0)=1$, and $\varepsilon^{\prime}(\nu)>0$.

The case $\varepsilon^{\prime} \geq 0$ is the normal case, since a higher meeting efficiency in this case increases a stayer's meeting probability as well as a visitor's. In this normal case, Proposition 3.3 shows that an increase in the meeting efficiency widens the price spread. More precisely, the highest price falls by less than the lowest price when the meeting efficiency increases. Regardless of whether $\varepsilon^{\prime} \geq 0$, an increase in $A$ increases the coefficient of variation in posted and transaction prices. The reason is similar to why a higher $x$ widens price dispersion in Corollary 3.1. By increasing visitors' meeting rate, a higher $A$ reduces a stayer's trading probability by more at high prices than at low prices. To maintain the same expected surplus in a meeting for all posted prices, as required by the equilibrium, the highest price must fall by less than the lowest price, resulting in wider dispersion in prices.

It is remarkable that an increase in $A$ can widen price dispersion. Because a higher meeting efficiency enables a visitor to meet with more stayers, one would think that the intensified competition among stayers would depress prices and narrow price dispersion. In the normal case $\varepsilon^{\prime} \geq 0$, an increase in $A$ does reduce prices, but it increases price dispersion. This result helps explain some puzzling findings on online price dispersion, e.g., Baye et al. (2004), Ellison and Ellison (2005). The Internet has increased the meeting efficiency substantially relative to the traditional search method, but it has not reduced price dispersion significantly. Baye et al. (2004) assembled a dataset containing millions of price observations in an online market for consumer electronics products in eight months from 2000 to 2001. The magnitude of price dispersion is comparable with that in the offline market. Moreover, although the usage of price comparison sites increased by about $13 \%$ in the period, there was no statistical evidence of any decline in price dispersion. However, they found that an increase in the number of sellers reduced price dispersion significantly. In the current model, a reduction in the entry cost $k$ increases the measure of stayers. It 
can be verified that $x^{*}$ falls and, by Corollary 3.1, price dispersion narrows. ${ }^{14}$

\section{Endogenous Search Effort and Efficient Policies}

This section endogenizes visitors' search effort. I show that price dispersion can induce search effort to be strategic complements among visitors. I re-examine social efficiency of the SMSE and recommend policies for managing aggregate activities efficiently.

\subsection{Equilibrium with endogenous search effort}

A visitor chooses search effort, $s$, after entering a submarket. Let $\psi(s)$ be the cost of search effort, with $\psi^{\prime}>0$ and $\psi^{\prime \prime}>0$ for all $s>0$, and $\psi^{\prime}(0)=0$. Let the meeting rate for a visitor be $x=\left(s+s_{0}\right) z$, where $z$ denotes the meeting rate per search effort. The constant $s_{0}>0$ rules out the uninteresting case where the equilibrium is stuck at $z=s=0 .{ }^{15}$ The total measure of visitors' search effort in a submarket is $\left(s+s_{0}\right) N_{v}$, and the measure of meetings in the submarket is $M\left(N_{s},\left(s+s_{0}\right) N_{v}\right)$. Since the total measure of meetings in the period is also equal to $x N_{v}$, then

$$
z=\frac{M\left(N_{s},\left(s+s_{0}\right) N_{v}\right)}{\left(s+s_{0}\right) N_{v}}=M(\theta, 1),
$$

where $\theta=\frac{N_{s}}{\left(s+s_{0}\right) N_{v}}$ is the effective tightness of the submarket. The above equation solves $\theta=\theta(z)$, which is a function of $z$ instead of $x$. The meeting probability for a stayer in a period is $\lambda(z)=\frac{z}{\theta(z)}$. Define $\bar{z} \leq \infty$ by $\theta(\bar{z})=\infty$. Submarkets are now described by $\left(p_{H}, z\right)$, instead of $\left(p_{H}, x\right)$. As a result, competitive entry of stayers into the market yields a stayer's expected surplus conditional on having a meeting as $\pi(z)=\frac{k}{\lambda(z)}$, which is a function of $z$ instead of $x$.

For stayers, the formulas in the baseline model remain valid after replacing $\pi(x)$ by $\pi(z)$ and $F(p \mid x)$ by $F(p \mid z)$. In a meeting, a stayer posting $p$ succeeds in trade with the

\footnotetext{
${ }^{14}$ In a different model, Bethune et al. (2018) show that a reduction in the cost of becoming informed of prices can increase price dispersion.

${ }^{15}$ If $s_{0}=0$, a visitor's meeting rate is $s z$, in which case the marginal gain to increasing $s$ is zero if $z=0$ and the marginal gain to increasing $z$ is also zero if $s=0$. Even a small $s_{0}>0$ can prevent this uninteresting case from happening in the equilibrium.
} 
probability $e^{-x F(p \mid z)}$. The stayer's expected surplus in the meeting is $p e^{-x F(p \mid z)}$. Equating this expected surplus to $\pi(z)$ for all prices on the support of $F$ yields:

$$
F(p \mid z)=\frac{1}{x} \ln \frac{p}{\pi(z)} .
$$

The inverse of this distribution function is:

$$
p=\pi(z) e^{x F}
$$

The bounds on the support of $F$ are:

$$
p_{L}=\pi(z), \quad p_{H}=\pi(z) e^{x}
$$

To analyze a visitor's optimal choices, suppose that one visitor searches with effort $\tilde{s}$ while all other visitors search with effort $s$. For the deviating visitor, the meeting rate is $\tilde{x} \equiv\left(\tilde{s}+s_{0}\right) z$, and the probability of trading at a price no higher than $p$ is $1-e^{-\tilde{x} F(p \mid z)}$. The deviating visitor's expected surplus in the submarket is:

$$
D(z, \tilde{s}) \equiv-\psi(\tilde{s})+\int_{p_{L}}^{p_{H}}(y-p-b) \mathrm{d}\left[1-e^{-\tilde{x} F(p \mid z)}\right] .
$$

This modifies (2.4) by subtracting search cost and changing the meeting rate to $\tilde{x}$. Note that stayers make their decisions based on the expectation that almost all visitors in the submarket will search with effort $s$. That is, $p$ is given by (4.2) where search effort is $s$ instead of $\tilde{s}$. Substituting such $p$, integrating and substituting $\pi$ from (2.9), I have:

$$
D(z, \tilde{s})=-\psi(\tilde{s})+(y-b)\left[1-e^{-\tilde{x}}\right]-k \theta(z)\left(\tilde{s}+s_{0}\right) L((\tilde{s}-s) z)
$$

where $\tilde{x}=\left(\tilde{s}+s_{0}\right) z$ and

$$
L(t) \equiv \frac{1-e^{-t}}{t} \text { for all }|t|<\infty .
$$

The visitor chooses $(z, \tilde{s})$ to maximize $D(z, \tilde{s})$.

To interpret the term $L((\tilde{s}-s) z)$, note that the last term in (4.5) is equal to $\tilde{x} L \pi$. As in the explanation for (2.10), this term is the surplus that the deviating visitor expects to concede to a stayer in the submarket. Thus, $L \pi$ is the concession normalized by the expected number of meetings for the visitor. If the visitor chooses the same search effort as other visitors do, this normalized concession is $L(0) \pi=\pi$. The following lemma lists the properties of $L$ (see the Supplementary Appendix C for a proof): 
Lemma 4.1. The function $L(t)$ defined by (4.6) has the following properties: (i) $L(0)=1$, $L^{\prime}(0)=-\frac{1}{2}$, and $L^{\prime \prime}(0)=\frac{1}{3}$; (ii) $L(t)>0, L^{\prime}(t)<0$ and $L^{\prime \prime}(t)>0$ for all $|t|<\infty$.

These properties are intuitive. If the deviating visitor searches more intensively than other visitors do, the visitor expects to be more likely to encounter a meeting with a lower price. As a result, the visitor expects to concede less than $\pi$ to a stayer in a meeting. Conversely, if the visitor searches less intensively than other visitors, the visitor expects to concede more than $\pi$ to a stayer in a meeting. Generally, a marginal increase in search effort reduces the visitor's expected concession by $-L^{\prime} \pi$. Since $L^{\prime}<0$, these expected "savings" for the deviating visitor are positive. However, this reduction in the expected concession diminishes as the visitor keeps increasing search effort, because $-L^{\prime \prime}<0$.

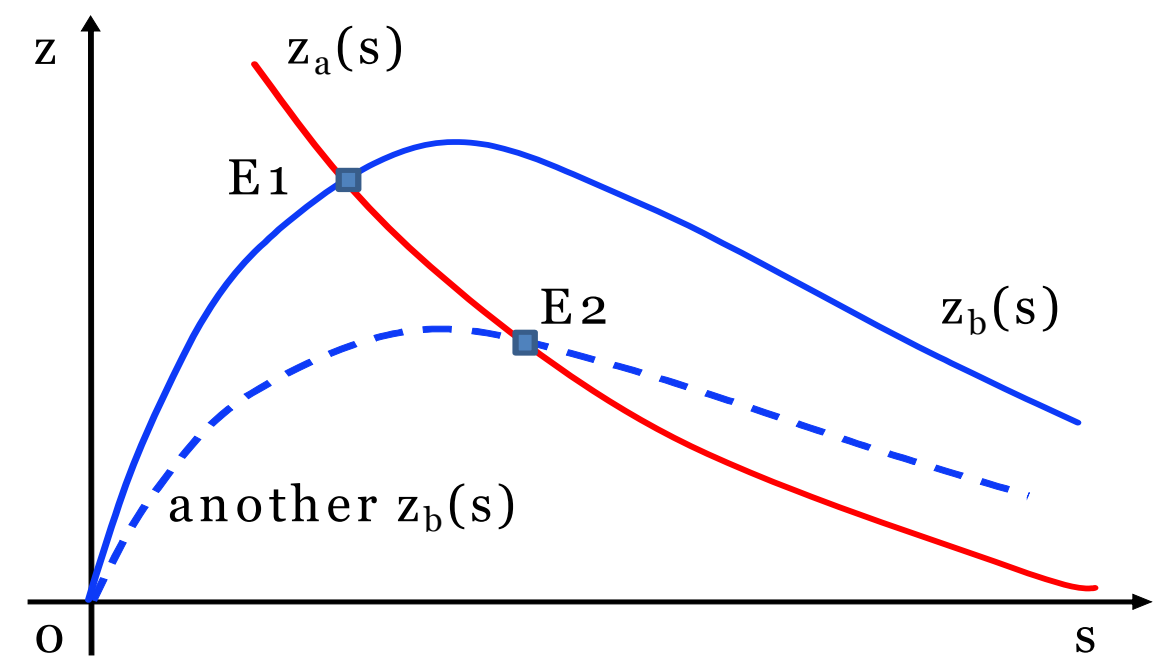

Figure 1. The unique equilibrium with endogenous search effort

An SMSE can be defined similarly to that in section 2.1 by adding search effort $\tilde{s}$ to a visitor's choice. I focus on symmetric equilibria, where $\tilde{s}=s$. As shown in Proposition 4.2 below, the optimal choices $(z, \tilde{s})$ are interior and satisfy the first-order conditions:

$$
\begin{gathered}
0=(y-b) e^{-\tilde{x}}-k\left[\theta^{\prime} L+(\tilde{s}-s) \theta L^{\prime}\right] \\
0=-\psi^{\prime}+(y-b) z e^{-\tilde{x}}-k \theta(z)\left[L+\tilde{x} L^{\prime}\right] .
\end{gathered}
$$

Denote the optimal choice of $z$ as $z^{*}$. In any symmetric SMSE, these conditions become:

$$
\begin{aligned}
& \theta^{\prime}(z)=\frac{y-b}{k} e^{-x} \\
& \psi^{\prime}(s)=k \theta(z)\left[\varepsilon(z)-1+\frac{x}{2}\right] .
\end{aligned}
$$


Recall that $x=\left(s+s_{0}\right) z$. Given any $s$, denote $z_{a}(s)$ as the solution for $z$ to the first equation in (4.9) and $z_{b}(s)$ as the solution for $z$ to the second equation. Figure 1 depicts $z_{a}(s)$ and two possibilities of $z_{b}(s)$. The SMSE is depicted by points E1 and E2, respectively, for the two possibilities of $z_{b}(s)$. The SMSE is unique in each case.

Formally, for any given $(s, z)$, denote a visitor's the optimal choice of $\tilde{s}$ as $S(s, z)$. The following proposition holds (see Appendix A for a proof):

Proposition 4.2. In all symmetric equilibria, the optimal choices $(z, \tilde{s})$ satisfy (4.7) and (4.8). A unique symmetric SMSE exists. In the SMSE, $S_{1}\left(s, z^{*}\right)<1$. Moreover, $S_{1}\left(s, z^{*}\right)>0$ if and only if $x^{*} \equiv\left(s+s_{0}\right) z^{*}>\frac{3}{2}$. An exogenous reduction in $\psi^{\prime}$ reduces $z$, increases $(s, x)$, and increases $\left(c v_{F}, c v_{G}\right)$. If $\varepsilon^{\prime} \geq 0$, this reduction in search cost reduces posted and transaction prices in the first-order stochastic dominance in $F$ and $G$.

The feature $S_{1}\left(s, z^{*}\right)>0$ means that search effort is "strategic complements" among visitors. Higher search effort by some visitors widens price dispersion and, thereby, increases the return on search for other visitors. For any given $z$, higher search effort by some visitors increases their meeting rates, $x$, and pushes prices down. However, the negative effect on prices is not uniform on all stayers. As explained for Corollary 3.1, the increase in $x$ reduces a stayer's trading probability by more at high prices than at low prices. To keep the expected surplus to be equal for all stayers, the highest price falls by less than the lowest price does. Thus, price dispersion increases, which motivates other visitors to increase search effort in order to find lower prices. Countering this positive effect of price dispersion on search effort, the reduction in price levels reduces visitors' incentive to search. The effect of price dispersion dominates if and only if price is sufficiently dispersed. This occurs when search effort is higher than a threshold $(3 / 2) \cdot{ }^{16}$

Although search effort can be complements among visitors, it is not strong complements, as shown by $S_{1}\left(s, z^{*}\right)<1$. A visitor's response in search effort to other visitors' search effort is limited by two forces. One is that the reduction in price levels reduces a visitor's

\footnotetext{
${ }^{16}$ This condition can be expressed as a restriction on the parameters. Since $x$ is an increasing function of $y, x$ is above the threshold $3 / 2$ if $y$ is above some threshold.
} 
gain from searching, as discussed above. The other is the equilibrium effect that higher search effort turns to reduce $z$. Since search effort and $z$ both increase a visitor's meeting rate, they are substitutes to a visitor. To induce a stayer to enter the market, a visitor expects to concede part of the match surplus to the stayer. This expected concession increases in $z$, because a higher $z$ implies a lower meeting rate for a stayer. When it is optimal for a visitor to increase search effort to increase the meeting rate, it is also optimal for the visitor to lower $z$ so as to reduce the expected concession to the stayer in a meeting. For any given $s$, a lower $z$ mitigate the increase in the meeting rate for a visitor, which limits the response of a visitor's search effort to other visitors' search effort.

Turning to an exogenous reduction in the marginal cost of search, I use Figure 1 to illustrate the effects. Before the reduction in search cost, let $z_{b}(s)$ be the solid curve in Figure 1. A reduction in search cost shifts the curve $z_{b}(s)$ to the dashed curve, which results in a higher $s$ and a lower $z$. It is intuitive that a reduction is search cost induces a visitor to search with higher effort and leads to a higher meeting rate for a visitor. As the matching probability for a stayer falls, a smaller measure of stayers enter the market, resulting in a fall in $z$. Under the mild condition $\varepsilon^{\prime} \geq 0$, posted and transaction prices fall in the first-order stochastic dominance. However, because $x$ increases, dispersion widens in both posted and transaction prices. The explanation for this result is similar to that in Corollary 3.1 for the effect of $x$ on price dispersion.

\subsection{Social inefficiency and corrective policies}

The complementarity in search effort raises the question whether the SMSE is socially efficient. To address this question, I incorporate visitors' search effort into the social planner's problem in section 3.2. The total cost of stayers' entry is $k N_{s}$. Because $N_{s}=$ $\theta(z)\left(s+s_{0}\right) N_{v}$, the entry cost divided by the measure of visitors is $k \theta(z)\left(s+s_{0}\right)$. Thus, social welfare normalized by the fixed measure of visitors is:

$$
-\psi(s)+(y-b)\left(1-e^{-x}\right)-k \theta(z)\left(s+s_{0}\right) .
$$


Maximizing social welfare, the planner's choices $(z, s)$ satisfies the first-order conditions:

$$
\theta^{\prime}(z)=\frac{y-b}{k} e^{-x}, \quad \psi^{\prime}(s)=k \theta(z)[\varepsilon(z)-1] .
$$

Compare these conditions with the counterparts in the SMSE, (4.9). The first condition is the same as in the SMSE. The second condition, which characterizes the socially efficient $s$, differs from that in the SMSE in the absence of the term $\frac{x}{2}$ on the right-hand side. This difference implies the following proposition (see Appendix A for a proof):

Proposition 4.3. Relative to the social optimum, the SMSE has excessive search effort $s$, a deficient $z$, and an excessive meeting rate for a visitor, $x$.

The inefficiency arises from an externality created by visitors' search effort. There is a private benefit for a visitor to increase search effort $\tilde{s}$ above others visitors' unexpectedly. Near $\tilde{s}=s$, this private benefit is: $-\pi x L^{\prime}(0)=\pi x / 2$. But if all visitors increase search effort, congestion increases, which is a negative externality. In the SMSE, search effort and the meeting rate for a visitor are inefficiently high. To maintain the optimal tradeoff between the meeting rate and the surplus of a trade, visitors choose an inefficiently low $z$. This low $z$ mitigates, but does not correct completely, the excessive $x$.

Two remarks follow. First, the inefficiency contrasts with models that have shown directed search to be able to internalize various externalities (e.g., Moen, 1997, Shi, 2001). To see why the contrast occurs, suppose that visitors can choose search effort before entering the market and commit to it. In that case, submarkets can be indexed by $\left(p_{H}, z, s\right)$, and visitors' optimal choice of the submarket will deliver the socially efficient outcome. Thus, the inability to commit to $s$ or to offer prices contingent on $s$ is critical for the inefficiency. This inability is realistic, and it is built into the timing that visitors choose search effort after entering a submarket. Second, noisy matching is important for the inefficiency, because a visitor increases search effort in order to find a lower price. If search is purely directed with a degenerate price distribution, as in Burdett et al. (2001), then endogenizing search effort does not generate inefficiency. Similarly, endogenizing search effort in models of competing auctions, as in Julien et al. (2000), does not make the equilibrium inefficient 
even though transaction prices are dispersed. The importance of noisy matching for the inefficiency provides a counter argument against the claim by Kennes et al. (2018) that different models of directed search are equivalent in terms of social welfare. ${ }^{17}$

To restore efficiency, consider the following combination of policies: a subsidy rate $\sigma_{e}$ to the entry cost, a lump-sum subsidy $\sigma_{n}$ to a visitor's participation in the market, and a proportional tax rate $\tau_{y}$ on the joint value of a trade. ${ }^{18}$ These policies maintain a balanced budget for the government. With the subsidy $\sigma_{e}$, competitive entry of stayers into the submarket pushes a stayer's expected surplus conditional on having a meeting to:

$$
\pi(z)=\frac{\left(1-\sigma_{e}\right) k}{\lambda(z)} .
$$

With this modified formula of $\pi(z)$, the inverse of the distribution of posted prices is still given by (4.2). A visitor's expected surplus in the market is given by (4.4), with $y$ being replaced by $\left(1-\tau_{y}\right) y$ and $\psi$ by $\psi-\sigma_{n}$. This expected surplus can be computed as

$$
\begin{aligned}
D(z, \tilde{s})= & -\psi(\tilde{s})+\sigma_{n}+\left[\left(1-\tau_{y}\right) y-b\right]\left[1-e^{-\tilde{x}}\right] \\
& -\left(1-\sigma_{e}\right) k \theta(z)\left(\tilde{s}+s_{0}\right) L((\tilde{s}-s) z) .
\end{aligned}
$$

In the symmetric SMSE, the first-order conditions of $(z, \tilde{s})$ become:

$$
\begin{aligned}
& \theta^{\prime}(z)=\frac{\left(1-\tau_{y}\right) y-b}{\left(1-\sigma_{e}\right) k} e^{-x} \\
& \psi^{\prime}(s)=\left(1-\sigma_{e}\right) k \theta(z)\left[\varepsilon(z)-1+\frac{x}{2}\right] .
\end{aligned}
$$

Comparing these conditions with (4.10) for the social optimum, it is easy to verify that the two sets of conditions coincide if and only if

$$
\sigma_{e}=\left[\frac{2[\varepsilon(z)-1]}{x}+1\right]^{-1}, \quad \tau_{y}=\sigma_{e}\left(1-\frac{b}{y}\right),
$$

where $(z, s)$ are the quantities in the social optimum. It is evident that $\sigma_{e}$ and $\tau_{y}$ lie in $(0,1)$. Because the measure of matches is $1-e^{-x}$, total tax revenue on the joint output

\footnotetext{
${ }^{17}$ Kennes et al. (2018) do not formulate SMS explicitly. They compare different matching models, one of which resembles SMS. I became aware of their paper after completing the first draft of the current paper. My paper formulates SMS explicitly and has different objectives. Moreover, I assume a general meeting function in contrast to the urn-ball matching function in their model, conduct comparative statics with respect to the meeting efficiency, endogenize search effort, and analyze corrective policies.

${ }^{18}$ A tax on visitors' search effort can also restore efficiency, but it requires the authority to have the unrealistic ability of observing visitors' search effort.
} 
across matches is $y \tau_{y}\left[1-e^{-x}\right]$. Total subsidy to the entry of stayers is $\sigma_{e} k\left(s+s_{0}\right) \theta$. The difference between the two is the lump-sum rebate to a visitor's participation in the market that balances the government budget. That is,

$$
\sigma_{n}=\sigma_{e}\left[\left(e^{x}-1\right) \varepsilon-x\right] \frac{k \theta}{z} .
$$

Since $e^{x}-1>x$ for all $x>0$ and since $\varepsilon>1$, then $\sigma_{n}(>0)$ is indeed a subsidy. With the above $\left(\sigma_{e}, \tau_{y}, \sigma_{n}\right)$, it can be verified that $D$ in the SMSE is equal to social welfare. The following proposition holds (see Appendix B for a proof):

Proposition 4.4. Suppose that $\left(\sigma_{e}, \sigma_{n}, \tau_{y}\right)$ satisfy (4.13)-(4.14). (i) The policies induce the equilibrium allocation $(z, s)$ and welfare to be socially efficient. (ii) The efficient policies increase $z$ and reduce the following variables: $s, x$, aggregate output $Y=y\left[1-e^{-x}\right]$, and $\left(c v_{F}, c v_{G}\right)$. (iii) If $\varepsilon^{\prime}(z) \geq 0$, the efficient policies reduce $\pi(z)$, reduce $\left(p_{L}, p_{H}, \Delta p\right)$, and reduce prices in the first-order stochastic dominance in $F(p \mid z)$ and $G(p \mid z)$.

With the subsidy to a stayer's entry, a stayer can accept a smaller expected concession from a visitor in a meeting and can still break-even on entry. A visitor's incentive to search weakens, which improves efficiency. However, the subsidy to the entry of stayers can also distort a visitor's tradeoff between the match surplus and the meeting rate. Given search effort, this tradeoff is efficient in the absence of the subsidy $\sigma_{e}$. The subsidy $\sigma_{e}$ induces a visitor to choose to enter a submarket that may have an inefficiently high $z$. A tax on the joint output in a match, $\tau_{y}$, can eliminate this distortion in a visitor's tradeoff by increasing the importance of the match surplus relative to the meeting rate for a visitor. When the subsidy $\sigma_{e}$ and the tax $\tau_{y}$ are set in such a way, the equilibrium allocation $(z, s)$ becomes socially efficient. The tax on the joint output generates a higher revenue than the subsidy to the entry cost. The surplus is rebated to visitors for participating in the market to balance the government budget.

The policies have two opposing effects on a stayer's expected surplus conditional on having a meeting, $\pi(z)$, which can be seen from (4.11). The subsidy to the entry cost reduces a stayer's effective cost of entry, $\left(1-\sigma_{e}\right) k$, which directly reduces $\pi$ needed for 
a stayer to break-even on entry. The indirect effect of the policies is that they reduce a stayer's meeting probability $\lambda(z)$, which requires $\pi$ to increase in order for a stayer to break-even on entry. The strength of the indirect effect relative to the direct effect depends on how sensitively $z$ responds to the policies. This sensitivity is regulated by $\varepsilon^{\prime}$, as explained for Proposition 3.3. The mild condition $\varepsilon^{\prime} \geq 0$ is sufficient for the policies to decrease $\pi$, and the condition is satisfied by widely used meeting functions in Example 3.4. If $\varepsilon^{\prime} \geq 0$, the marginal cost of increasing $z$, in terms of $\theta^{\prime}(z)$, increases sharply in $z$. As a result, $z$ and $\lambda(z)$ respond to the policies by only a small amount in the SMSE. The direct effect of the policies through a stayer's effective entry cost dominates the indirect effect to reduce $\pi(z)$. The indirect effect of the policies through $\lambda(z)$ can dominate the direct effect to increase $\pi(z)$ only when $\varepsilon^{\prime}$ is sufficiently negative.

The response of $\pi$ to the policies is important for the responses of prices. If the policies reduce $\pi$, then a stayer can achieve $\pi$ with lower prices. In addition, because the policies reduce a visitor meeting rate $x$, a stayer faces a lower risk of losing a visitor to a competitor. This further reduces prices that are needed to achieve $\pi$. Thus, if $\varepsilon^{\prime} \geq 0$, the policies reduce posted and transaction prices according to the first-order stochastic dominance. The reduced competition benefits a stayer who posts the highest price by more than a stayer who posts a lower price. The price spread narrows. The condition $\varepsilon^{\prime} \geq 0$ is sufficient for prices to respond in this way. Even if $\varepsilon^{\prime}$ is sufficiently negative to make $\pi$ increase in response to the policies, the policies may still reduce prices and the price spread. Regardless of whether $\varepsilon^{\prime} \geq 0$, the efficient policies reduce the coefficient of variation, in both posted prices and transaction prices.

The corrective policies depend on the features of the SMSE intuitively. In particular, the subsidy to stayers' entry cost is higher if visitors' search effort is higher, because the subsidy is used to reduce the inefficiently high search effort. Also, the tax on the joint value of a trade higher if the subsidy to a stayer's entry is higher, because the tax is used to correct the consequence of the entry subsidy for a visitor's choice of the submarket. 


\subsection{Management of aggregate activities}

The corrective policies can also manage aggregate activities efficiently when the aggregate economic condition changes. I focus on $y$ as an indicator of the aggregate condition. Because $y$ is the joint value of a trade, it can reflect both the aggregate supply condition, such as aggregate productivity, and the aggregate demand condition, such as changes in preferences. I refer to a higher $y$ as a better economic condition.

In Figure 1, an increase in $y$ shifts up the curve $z_{a}(s)$ and leaves the curve $z_{b}(s)$ intact. Equilibrium $s$ increases. Equilibrium $z$ increases if the SMSE is one like point E1, but decreases if the SMSE is one like point E2. Formally, the following proposition states how the SMSE and the corrective policies respond to $y$ (see Appendix B for a proof):

Proposition 4.5. Without the corrective policies, an increase in $y$ has the following effects on the SMSE: $\frac{d s}{d y}>0, \frac{d x}{d y}>0, \frac{d p_{H}}{d y}>0, \frac{d \Delta p}{d y}>0, \frac{d}{d y} c v_{F}>0$, and $\frac{d}{d y} c v_{G}>0$. If $\psi^{\prime \prime \prime}>0$, then $\frac{d z}{d y}>0$. If $\psi^{\prime \prime \prime}<0$, then there exists $s_{a} \in(0, \infty]$ such that $\frac{d z}{d y}>0$ iff $s<s_{a}$. With the efficient policies in (4.13), a sufficient condition for $\frac{d \sigma_{e}}{d y}>0$ is $\varepsilon^{\prime}(z) \leq(\varepsilon-1) / z$.

By increasing the gains from a match, a higher $y$ increases the return on search and, hence, increases search effort and the meeting for a visitor, $x$. As explained for Corollary 3.1 , a higher $x$ widens price dispersion, as measured by the spread $\Delta p$ and coefficients of variation $\left(c v_{F}, c v_{G}\right)$. However, $z$ responds to a higher $y$ ambiguously. Because a higher $y$ induces search effort to increase, the surplus that a visitor expects to concede to a stayer in a meeting increases, as explained above. To restrict the increase in this concession, a visitor may reduce $z$. This happens when search effort is high. When search effort is low, the direct effect of $y$ on $z$ dominates, in which case $z$ increases.

Without the policies, an improvement in the economic condition results in an overheated economy. Search effort and a visitor's meeting rate increase by an excessive amount, and $z$ falls by too much. The inefficiently higher search effort of visitors results in inflated prices and inefficiently wide dispersion in prices. To manage aggregate activities efficiently, the corrective policies should cool down the economy. As Proposition 4.4 shows, this can 
be achieved by the combination of a subsidy to stayers' entry and a tax on the joint output. These corrective policies tame visitors' search effort, increase $z$, and moderate the increase in aggregate output. Under the mild condition $\varepsilon^{\prime} \geq 0$, these policies also moderate the increase in prices and prevent price dispersion from widening excessively.

The policies are automatic stabilizers for the economy even if the subsidy rate to stayers' entry and the tax on the joint value of a trade are constant. However, social efficiency may call for these policies to be proactive in the sense that they should increase when the economic condition improves. As stated in Proposition 4.5, a sufficient condition for these policies to be proactive is $\varepsilon^{\prime} \leq(\varepsilon-1) / z$. By the explanation for the response of $\pi$ to the policies in Proposition 4.4, a large $\varepsilon^{\prime}$ implies that the equilibrium $z$ is insensitive to changes in the economic condition. Thus, unless the equilibrium $z$ is very insensitive to changes in the economic condition, the subsidy to stayers' entry and the tax on the joint value of a trade should increase in an economic boom, and should decrease in a recession.

The corrective policies contrast with those in Diamond (1982), who assumes the matching function to have increasing returns to scale. Because of this assumption, aggregate demand can be inefficiently low, which can be stimulated with policies. In the current model, the meeting and the matching functions have constant returns to scale. Visitors' choices of search effort generate an externality that leads to an overheated economy. The policies are recommended to cool down the economy. Also, instead of managing aggregate demand, the recommended policies manage both aggregate demand and supply in the current model.

\section{Conclusion}

This paper has analyzed sequentially mixed search (SMS), which consists of directed search in the first stage and noisy matching with multiple offers in the second stage. I establish existence of a unique equilibrium (SMSE), and analyze how the two stages of SMS interact to affect quantities and price dispersion. Comparative statics demonstrate how the SMSE responds to changes in the economic condition and the meeting efficiency. By extending the model to endogenize search effort, I show that search effort, aggregate output and price 
dispersion are all excessively high in the SMSE relative to the constrained social optimum. Policies are introduced to restore efficiency and manage aggregate demand and supply.

The simple framework of SMS in this paper opens up many possibilities for future research. In a sequel (Shi, 2018), I study the SMSE in the environment with many-to-many meetings where both stayers and visitors can have more than one meeting. Let me mention two additional research directions. The first is to introduce heterogeneity. If visitors have ex ante heterogeneity that affects the tradeoff between $p_{H}$ and $x$, different visitors will selfselect into different submarkets. If heterogeneity is realized after visitors enter a submarket, then it will affect stayers' pricing strategy. Both types of heterogeneity may be relevant in a typical market such as the labor market. The second direction for future research is to incorporate trading relationships and business cycles. In the goods market, past trades create customer relationships, as analyzed in Gourio and Rudanko (2014) and Shi (2016). In the labor market, employment is a lasting relationship between a firm and a worker, as analyzed by Burdett and Mortensen (1998). In both markets, individuals in a relationship can continue to search. By embedding search on the relationship into a business cycle model, the analysis can shed new light on how the turnover of relationships and the price distribution fluctuate in the business cycle. Directed search in the first stage of SMS will be important for such an analysis to be tractable by making the SMSE block recursive, as formulated by Shi (2009) and Menzio and Shi (2010). 


\section{Appendix}

\section{A. Proofs for Propositions 4.2 and 4.3}

\section{Proof of Proposition 4.2:}

First, I prove that a visitor's optimal choices $(z, \tilde{s})$ are interior and satisfy $(4.7)$ and (4.8). Because $D(z, \tilde{s})$ is differentiable in $(z, \tilde{s})$, the optimal choices satisfy the first-order conditions if they are interior. The derivative $D_{1}(z, \tilde{s})$ is equal to the right-hand side of (4.7) multiplied by $\left(\tilde{s}+s_{0}\right)$. Since $\theta(0)=0, L(0)=1$ and $L^{\prime}(0)=-\frac{1}{2}$, then

$$
D_{1}(0, \tilde{s})=\left(\tilde{s}+s_{0}\right)\left[y-b-k \theta^{\prime}(0)\right]>0 \text { for all } \tilde{s} \geq 0
$$

where the inequality is a maintained assumption. Similarly, $D_{1}(\infty, \tilde{s})<0$. Thus, for all $\tilde{s}>0$, the optimal $z$ is interior and satisfies the first-order condition $(4.7) . D_{2}(z, \tilde{s})$ is given by the right-hand side of (4.8). In any symmetric SMSE, I have:

$$
D_{2}(z, s)=-\psi^{\prime}+(y-b) z e^{-x}-k \theta\left[1-\frac{x}{2}\right] .
$$

Substituting $(y-b) e^{-x}$ from the version of (4.7) in the symmetric SMSE, I get:

$$
D_{2}(z, s)=-\psi^{\prime}+k \theta\left[\varepsilon-1+\frac{x}{2}\right]
$$

Thus $D_{2}(z, 0)>0$ for $z>0$. That is, in any symmetric SMSE, $\tilde{s}=s>0$. Also, $D_{2}(z, \infty)<0$, and so the optimal $\tilde{s}$ satisfies $\tilde{s}<\infty$. Thus, in any symmetric SMSE, the optimal $\tilde{s}$ is interior and satisfies the first-order condition (4.8).

Second, I prove that a unique SMSE exists. Recall that, given any $s, z_{a}(s)$ denotes the solution for $z$ to the first equation in (4.9) and $z_{b}(s)$ the solution to the second equation. The equilibrium value of $s$ solves $z_{b}(s)-z_{a}(s)=0$. The assumption $y-b>k \theta^{\prime}(0)$ implies $z_{a}(0)>0$. Also, $z_{a}(\infty)=0$. Since $\psi^{\prime}(0)=0$ and $\theta(0)=0$, then $z_{b}(0)=0<z_{a}(0)$ and $z_{b}(\infty)>0=z_{a}(\infty)$. Thus, the equation $z_{b}(s)=z_{a}(s)$ has at least one solution in $(0, \infty)$. To prove that the solution is unique, compute:

$$
z_{a}^{\prime}(s)=\frac{-z}{\frac{\theta^{\prime \prime}}{\theta^{\prime}}+s+s_{0}}<0, \quad z_{b}^{\prime}(s)=\frac{\psi^{\prime \prime}-k z \theta / 2}{k\left[(\varepsilon+1) \frac{s+s_{0}}{2}+z \theta^{\prime \prime}\right]} .
$$


Here I substituted $\varepsilon^{\prime}=\frac{z \theta^{\prime \prime}}{\theta}-\frac{\varepsilon(\varepsilon-1)}{z}$. The difference $\left[z_{b}^{\prime}\left(s_{0}\right)-z_{a}^{\prime}\left(s_{0}\right)\right]$ has the same sign as

$$
\left(\frac{\theta^{\prime \prime}}{\theta^{\prime}}+s+s_{0}\right) \psi^{\prime \prime}+k \theta\left[\frac{z \theta^{\prime \prime}}{\theta^{\prime}}\left(\varepsilon-\frac{1}{2}\right)+\varepsilon \frac{x}{2}\right] .
$$

Since this is positive, then $z_{b}^{\prime}(s)>z_{a}^{\prime}(s)$ for all $s \geq 0$. Thus, the solution to $z_{a}(s)=z_{b}(s)$ is unique, as multiple solutions necessarily have alternating signs of $\left[z_{b}^{\prime}(s)-z_{a}^{\prime}(s)\right]$.

Third, I prove $S_{1}\left(s, z^{*}\right)<1$ and find the condition for $S_{1}\left(s, z^{*}\right)>0$, where $S(s, z)$ is a visitor's optimal choice of $\tilde{s}$ given $(s, z)$. Differentiating $(4.8)$, I can compute:

$$
S_{1}(s, z)=\frac{k \theta(z) z}{-D_{22}(z, \tilde{s})}\left[L^{\prime}+\tilde{x} L^{\prime \prime}\right] .
$$

In the symmetric SMSE, $\tilde{s}=s$. Using the features of $L$ in Lemma 4.1, I can evaluate:

$$
\begin{aligned}
& D_{22}\left(z^{*}, s\right)=-\psi^{\prime \prime}-k z \theta[\varepsilon-1+x / 3]<0, \\
& S_{1}\left(s, z^{*}\right)=\frac{k \theta\left(z^{*}\right) z^{*}}{-D_{22}\left(z^{*}, s\right)}\left[\frac{x^{*}}{3}-\frac{1}{2}\right] .
\end{aligned}
$$

Here $z^{*}$ is used instead of $z$ because the first-order condition of $z,(4.7)$, is used to substitute the term $(y-b) e^{-\tilde{x}}$. Thus, $S_{1}\left(s, z^{*}\right)>0$ if and only if $x^{*}>\frac{3}{2}$. Moreover, $S_{1}\left(s, z^{*}\right)<1$ if and only if $\psi^{\prime \prime}+k z \theta\left(\varepsilon-\frac{1}{2}\right)>0$, which is satisfied.

Finally, suppose $\psi(s)=\psi_{0} \hat{\psi}(s)$ for some constant $\psi_{0}>0$ and a function $\hat{\psi}(s)$. An increase in $\psi_{0}$ is an exogenous increase in the marginal cost of search effort. A reduction in $\psi_{0}$ is an exogenous reduction in the marginal cost of search effort. Differentiating (4.9) with respect to $\psi_{0}$ shows $\frac{\mathrm{d} z}{\mathrm{~d} \psi_{0}}>0, \frac{\mathrm{d} s}{\mathrm{~d} \psi_{0}}<0$, and $\frac{\mathrm{d} x}{\mathrm{~d} \psi_{0}}<0$. Since $c v_{F}^{\prime}(x)>0$ and $c v_{G}^{\prime}(x)>0$ by Corollary 3.1, then $\frac{\mathrm{d}}{\mathrm{d} \psi_{0}} c v_{F}<0$ and $\frac{\mathrm{d}}{\mathrm{d} \psi_{0}} c v_{G}<0$. Similar to the proof of the effects of $\sigma_{e}$ later in Proposition 4.4, the increase in $z$ and the fall in $x$ imply that, if $\varepsilon^{\prime} \geq 0$, then $\frac{\mathrm{d} F}{\mathrm{~d} \psi_{0}}<0$ and $\frac{\mathrm{d} G}{\mathrm{~d} \psi_{0}}<0$. That is, an in $\psi_{0}$ increase prices in the first-order stochastic dominance in $F$ and $G$. QED

\section{Proof of Proposition 4.3:}

The first equation in (4.10) is identical to the first equation in (4.9). For any given $s$, the common solution for $z$ to the two equations is $z_{a}(s)$. Let $z_{b}^{e}(s)$ denote the solution for the equilibrium $z$ to the second equation in (4.9), and $z_{b}^{o}(s)$ the solution for the socially optimal $z$ to the second equation in (4.10). Denote $\Delta z^{e}(s)=z_{b}^{e}(s)-z_{a}(s)$ and $\Delta z^{o}(s)=$ 
$z_{b}^{o}(s)-z_{a}(s)$. Then, the equilibrium $s$ solves $\Delta z^{e}(s)=0$, and the socially optimal $s$ solves $\Delta z^{o}(s)=0$. As shown in the proof of Proposition 4.2, $\Delta z^{e^{\prime}}(s)>0$. Similarly, $\Delta z^{\prime \prime}(s)>0$. For any $s \geq 0$ and $z>0$, the right-hand side of the second condition in (4.9) is strictly greater than that of the second condition in (4.10). For any given $s \geq 0$, a smaller $z$ is required to satisfy the second equation in (4.9) than to satisfy the second equation in (4.10). That is, $z_{b}^{e}(s)<z_{b}^{o}(s)$ for all $s \geq 0$. This implies $\Delta z^{e}(s)<\Delta z^{o}(s)$ for all $s \geq 0$. Since $\Delta z^{e^{\prime}}(s)>0$ and $\Delta z^{o \prime}(s)>0$, the solution for $s$ to $\Delta z^{e}(s)=0$ must be strictly larger than the solution for $s$ to $\Delta z^{o}(s)=0$. Because $z$ satisfies the common equation $z=z_{a}(s)$ in the SMSE and the social optimum, and because $z_{a}^{\prime}(s)<0$, then $z$ is lower in the equilibrium $z$ than in the social optimum. Backing out $x$ from the common equation for $z_{a}(s)$ in the SMSE and in the social optimum, I conclude that $x$ is higher in the SMSE than in the social optimum. This completes the proof of Proposition 4.3. QED

\section{B. Proofs for Propositions 4.4 and 4.5}

\section{Proof of Proposition 4.4:}

(i) The text preceding the proposition has established the result (i) that the policies $\left(\sigma_{e}, \tau_{y}, \sigma_{n}\right)$ induce the SMSE to be socially efficient.

(ii) By Proposition 4.3, the SMSE without the policies has an excessive $s$, a deficient $z$ and an excessive $x$, relative to the social optimum. Because the efficient policies restore the social optimum, they reduce $s$, increase $z$ and reduce $x$.

(iii) I analyze the effects of the policies on prices in three steps. First, I reduce the dimension of the policies and variables. To do so, let $\tau_{y}$ depend on $\sigma_{e}$ as in (4.13). Then, for all $\sigma_{e}$, the first equation in (4.12) is the same as the first equation in (4.9), and the common solution for $z$ to the two equations is $z=z_{a}(s)$. For any given $s, z_{a}(s)$ does not depend on the policies directly. Also, $z_{a}^{\prime}(s)<0$, as shown in the proof of Proposition 4.2. Substituting $z=z_{a}(s)$ into the second equation of (4.12), I get:

$$
\psi^{\prime}(s)=\left(1-\sigma_{e}\right) k \theta(z)\left[\varepsilon(z)-1+\frac{x}{2}\right]_{z=z_{a}(s)} .
$$

Second, I compute the effects of $\sigma_{e}$ on $(s, z), Y$ and $\left(c v_{F}, c v_{G}\right)$, taking into account 
the dependence of $\tau_{y}$ on $\sigma_{e}$ in (4.13). In this computation, $\sigma_{e}$ is arbitrary instead of the efficient one. However, if the derivative of a variable with respect to $\sigma_{e}$ is positive, then the variable increases under the efficient policies, because the efficient policies have $\sigma_{e}>0$. Differentiating (B.1) with respect to $\sigma_{e}$, I get:

$$
\frac{\mathrm{d} s}{\mathrm{~d} \sigma_{e}}=\frac{-\psi^{\prime}}{1-\sigma_{e}}\left[\psi^{\prime \prime}+\frac{(2 \varepsilon-1) z \theta^{\prime \prime}+x \theta^{\prime} \varepsilon}{\theta^{\prime \prime}+\left(s+s_{0}\right) \theta^{\prime}} \frac{1-\sigma_{e}}{2} k \theta\right]^{-1}<0 .
$$

This implies $\frac{\mathrm{d} z}{\mathrm{~d} \sigma_{e}}=z_{a}^{\prime}(s) \frac{\mathrm{d} s}{\mathrm{~d} \sigma_{e}}>0$ and

$$
\frac{\mathrm{d} x}{\mathrm{~d} \sigma_{e}}=\frac{z \theta^{\prime \prime}}{\theta^{\prime \prime}+\left(s+s_{0}\right) \theta^{\prime}} \frac{\mathrm{d} s}{\mathrm{~d} \sigma_{e}}<0 .
$$

Since aggregate output is $Y=y\left[1-e^{-x}\right]$, it is clear that $\frac{\mathrm{d} Y}{\mathrm{~d} \sigma_{e}}<0$. The coefficients of variation in prices, $c v_{F}(x)$ and $c v_{G}(x)$, are given by (3.1). Since $c v_{F}^{\prime}(x)>0$ and $c v_{G}^{\prime}(x)>0$ by Corollary 3.1, and since $\frac{\mathrm{d} x}{\mathrm{~d} \sigma_{e}}<0$, then $\frac{\mathrm{d}}{\mathrm{d} \sigma_{e}} c v_{F}<0$ and $\frac{\mathrm{d}}{\mathrm{d} \sigma_{e}} c v_{G}<0$.

Third, I compute the effects of $\sigma_{e}$ on $\pi(z)$ and prices, again taking into account the dependence of $\tau_{y}$ on $\sigma_{e}$. With the policies, $\pi(z)=\frac{\left(1-\sigma_{e}\right) k}{\lambda(z)}$. Using the above results for $\frac{\mathrm{d} s}{\mathrm{~d} \sigma_{e}}$ and $\frac{\mathrm{d} z}{\mathrm{~d} \sigma_{e}}$, I compute:

$$
\begin{aligned}
& -\frac{\lambda}{k}\left\{\left[\theta^{\prime \prime}+\left(s+s_{0}\right) \theta^{\prime}\right] \psi^{\prime \prime}+\left[(2 \varepsilon-1) z \theta^{\prime \prime}+x \theta^{\prime} \varepsilon\right] \frac{1-\sigma_{e}}{2} k \theta\right\} \frac{\mathrm{d} \pi(z)}{\mathrm{d} \sigma_{e}} \\
& =\left[(2 \varepsilon-1) z \theta^{\prime \prime}+x \theta^{\prime} \varepsilon\right] \frac{1-\sigma_{e}}{2} k \theta-(\varepsilon-1) \theta^{\prime} \psi^{\prime}+\left[\theta^{\prime \prime}+\left(s+s_{0}\right) \theta^{\prime}\right] \psi^{\prime \prime} .
\end{aligned}
$$

For the right-hand side to be strictly positive, a sufficient condition is that the difference between the first two terms is non-negative. Substituting $\psi^{\prime}$ from (B.1) and substituting $z \theta^{\prime \prime}=\theta \varepsilon^{\prime}+(\varepsilon-1) \theta^{\prime}$, I rewrite this difference as:

$$
\frac{1-\sigma_{e}}{2} k \theta\left\{(2 \varepsilon-1) \theta \varepsilon^{\prime}+[\varepsilon-1+x] \theta^{\prime}\right\}
$$

Since $\varepsilon>1$, a sufficient condition for the above expression to be strictly positive is $\varepsilon^{\prime} \geq 0$. Thus, $\varepsilon^{\prime} \geq 0$ is sufficient for $\frac{\mathrm{d} \pi(z)}{\mathrm{d} \sigma_{e}}<0$.

Assume $\varepsilon^{\prime} \geq 0$. For any given $p,(4.1)$ implies:

$$
x \frac{\mathrm{d} F(p \mid z)}{\mathrm{d} \sigma_{e}}=-F \frac{\mathrm{d} x}{\mathrm{~d} \sigma_{e}}-\frac{\mathrm{d} \pi(z)}{\mathrm{d} \sigma_{e}}>0 .
$$

Thus, the policies reduce posted prices in the first-order stochastic dominance. Similar to (2.5), the distribution of transactions prices is:

$$
G(p \mid z) \equiv \frac{1-e^{-x F(p \mid z)}}{1-e^{-x}}=\frac{1-\frac{\pi(z)}{p}}{1-e^{-x}},
$$


where the second equality comes from substituting $F$ from (4.1). Because $\sigma_{e}$ reduces $\pi(z)$ and $x$, then $\frac{\mathrm{d} G(p \mid z)}{\mathrm{d} \sigma_{e}}>0$ for any given $p$. That is, the policies reduce transaction prices in the first-order stochastic dominance. Moreover, (4.2) implies:

$$
\frac{\mathrm{d} p_{L}}{\mathrm{~d} \sigma_{e}}=\frac{\mathrm{d} \pi(z)}{\mathrm{d} \sigma_{e}}<0, \frac{\mathrm{d} p_{H}}{\mathrm{~d} \sigma_{e}}=e^{x F}\left[\frac{\mathrm{d} \pi(z)}{\mathrm{d} \sigma_{e}}+\pi(z) F \frac{\mathrm{d} x}{\mathrm{~d} \sigma_{e}}\right]<0 .
$$

The policies affect the price spread, $\Delta p=p_{H}-p_{L}$, as follows:

$$
\frac{\mathrm{d} \Delta p}{\mathrm{~d} \sigma_{e}}=\left(e^{x F}-1\right) \frac{\mathrm{d} \pi(z)}{\mathrm{d} \sigma_{e}}+\pi(z) F e^{x F} \frac{\mathrm{d} x}{\mathrm{~d} \sigma_{e}}<0 .
$$

This completes the proof of Proposition 4.4. QED

\section{Proof of Proposition 4.5:}

Comparative statics with respect to $y$ without the policies: In the absence of the policies, the SMSE is characterized by (4.9). For any given $s$, the solution for $z$ is $z_{a}(s)$ to the first equation in (4.9) and $z_{b}(s)$ to the second equation. To prove $\frac{\mathrm{d} s}{\mathrm{~d} y}>0$, note that $\frac{\partial z_{a}(s)}{\partial y}>0$ and $\frac{\partial z_{b}(s)}{\partial y}=0$, where the partial derivatives are taken for any given $s$. Since $z_{b}^{\prime}(s)>z_{a}^{\prime}(s)$ with the optimal $s$, then

$$
\frac{\mathrm{d} s}{\mathrm{~d} y}=\frac{\partial z_{a}(s) / \partial y}{z_{b}^{\prime}(s)-z_{a}^{\prime}(s)}>0 .
$$

Because $\frac{\partial z_{b}(s)}{\partial y}=0$, then

$$
\frac{\mathrm{d} z}{\mathrm{~d} y}=z_{b}^{\prime}(s) \frac{\mathrm{d} s}{\mathrm{~d} y} .
$$

Therefore, the sign of $\frac{\mathrm{d} z}{\mathrm{~d} y}$ is the same as $z_{b}^{\prime}(s)$, which is ambiguous and examined below.

Similarly, using the notation $x=\left(s+s_{0}\right) z$ to express $s=\frac{x}{z}-s_{0}$, I can use the two equations in (4.9) to solve for $z=z_{a}(x)$ and $z=z_{b}(x)$. Then,

$$
z_{a}^{\prime}(x)=\frac{-\theta^{\prime}}{\theta^{\prime \prime}}, \quad z_{b}^{\prime}(x)=\frac{\psi^{\prime \prime}-\frac{k \theta}{2} z}{k z\left[z \theta^{\prime \prime}+\frac{x}{2} \theta^{\prime}\right]+\psi^{\prime \prime} \frac{x}{z}} .
$$

Again, it can be verified that $z_{b}^{\prime}(x)>z_{a}^{\prime}(x)$. Thus, there is a unique solution $x$ to $z_{b}(x)=$ $z_{a}(x)$, and the solution satisfies $\frac{\mathrm{d} x}{\mathrm{~d} y}>0$. Furthermore, $\frac{\mathrm{d}\left(\pi e^{x}\right)}{\mathrm{d} y}>0$, and so $\frac{\mathrm{d} p_{H}}{\mathrm{~d} y}>0$. If $\frac{\mathrm{d} z}{\mathrm{~d} y} \leq 0$, then $\frac{\mathrm{d} p_{L}}{\mathrm{~d} y} \leq 0$ and $\frac{\mathrm{d} \Delta p}{\mathrm{~d} y}>0$. If $\frac{\mathrm{d} z}{\mathrm{~d} y}>0$, then $\frac{\mathrm{d} \pi}{\mathrm{d} y}>0$ and

$$
\frac{\mathrm{d} \Delta p}{\mathrm{~d} y}=\left(e^{x}-1\right) \frac{\mathrm{d} \pi}{\mathrm{d} y}+\pi e^{x} \frac{\mathrm{d} x}{\mathrm{~d} y}>0 .
$$


In all cases, $\frac{\mathrm{d} \Delta p}{\mathrm{~d} y}>0$. Since $c v_{F}^{\prime}(x)>0$ and $c v_{G}^{\prime}(x)>0$, the result $\frac{\mathrm{d} x}{\mathrm{~d} y}>0$ implies $\frac{\mathrm{d}}{\mathrm{d} y} c v_{F}>0$ and $\frac{\mathrm{d}}{\mathrm{d} y} c v_{G}>0$.

Finally, I establish the sign of $z_{b}^{\prime}(s)$. Return to the use of $s$ instead of $x$ as the variable. Denote $z_{c}(s)$ as the solution to $\theta\left(z_{c}\right) z_{c}=2 \psi^{\prime \prime}(s) / k$. Then, $z_{b}^{\prime}(s)>0$ if and only if $z<z_{c}(s)$. Because $\psi^{\prime \prime}>0$, then $z_{c}\left(s_{0}\right)>0$. Consider the following cases:

Case (i): $\psi^{\prime \prime \prime}>0$. In this case, $z_{c}^{\prime}(s)>0$ for all $s$. I prove that $z_{b}^{\prime}(s)>0$ for all $s \geq s_{0}$, which implies $\frac{\mathrm{d} z}{\mathrm{~d} y}>0$ by the above proof. A sufficient condition for this result is $z_{b}(s)<z_{c}(s)$ for all $s \geq 0$. To prove that this sufficient condition holds, suppose, to the contrary, that $z_{b}\left(s_{a}\right)=z_{c}\left(s_{a}\right)$ for some $s_{a} \in[0, \infty)$. Clearly, $s_{a}>0$ and $z_{b}^{\prime}\left(s_{a}\right)=0$. Without loss of generality, let $s_{a}$ be the smallest solution to $z_{b}(s)=z_{c}(s)$. Because $z_{b}(s)<z_{c}(s)$ for all $s<s_{a}$, and $z_{b}\left(s_{a}\right)=z_{c}\left(s_{a}\right)$, then $z_{b}^{\prime}\left(s_{a}\right) \geq z_{c}^{\prime}\left(s_{a}\right)>0$. This contradicts the fact that $z_{b}^{\prime}\left(s_{a}\right)=0$. Thus, $s_{a}>0$ does not exist, and so $z_{b}(s)<z_{c}(s)$ for all $s>s_{0}$. In this case, the SMSE is depicted by point E1 in Figure 1.

Case (ii): $\psi^{\prime \prime \prime}<0$, but $z_{b}(s)<z_{c}(s)$ for all $s$. As in case (i), this case has $z_{b}^{\prime}(s)>0$ for all $s$, and so $\frac{\mathrm{d} z}{\mathrm{~d} y}>0$.

Case (iii): $\psi^{\prime \prime \prime}<0$, and there exists $s_{a}>0$ such that $z_{b}\left(s_{a}\right)=z_{c}\left(s_{a}\right)$. Let $s_{a}$ be the smallest solution to $z_{b}(s)=z_{c}(s)$. I prove that $s_{a}$ is the only solution to $z_{b}(s)=$ $z_{c}(s)$. Suppose, to the contrary, that there is another solution $s_{1}\left(>s_{a}\right)$ to $z_{b}(s)=$ $z_{c}(s)$. Without loss of generality, let $s_{1}$ be the smallest solution among all $s>s_{a}$. Then $z_{b}\left(s_{1}-\varepsilon\right)>z_{c}\left(s_{1}-\varepsilon\right)$ for sufficiently small $\varepsilon>0$. This fact and the definition of $s_{1}$ imply $z_{b}^{\prime}\left(s_{1}\right) \leq z_{c}^{\prime}\left(s_{1}\right)<0$. This contradicts the fact that $z_{b}^{\prime}\left(s_{1}\right)=0$. Thus, $s_{1}$ does not exist; i.e., $z_{b}(s)>z_{c}(s)$ for all $s>s_{a}$. Therefore, $z_{b}^{\prime}(s)<0$ if and only if $s>s_{a}$. That is, $\frac{\mathrm{d} z}{\mathrm{~d} y}>0$ if and only if the optimal $s$ satisfies $s<s_{a}$. In this case, the SMSE is depicted by point E2 in Figure 1.

Case (iv): $\psi^{\prime \prime \prime}=0$. In this case, $z_{c}(s)$ is constant over $s$. If $z_{b}(s)<z_{c}(s)$ for all $s>0$, the case is qualitatively the same as case (i). If there is a solution $s_{a}>0$ to $z_{b}(s)=z_{c}(s)$, then $z_{b}(s)=z_{c}(s)$ for all $s>s_{a}$. In this case, $\frac{\mathrm{d} z}{\mathrm{~d} y}>0$ if $s<s_{a}$, and $\frac{\mathrm{d} z}{\mathrm{~d} y}=0$ if $s>s_{a}$.

The effect of $y$ on the efficient policies: Under the efficient policies in (4.13), the 
equilibrium allocation coincides with the social optimum given by (4.10). Differentiating (4.10) with respect to $y$, I get:

$$
\frac{\mathrm{d} z}{\mathrm{~d} y}=\frac{e^{-x}}{k\left[\left(s+s_{0}\right) \theta^{\prime}+\left(\frac{z^{2} k \theta^{\prime}}{\psi^{\prime \prime}}+1\right) \theta^{\prime \prime}\right]}>0, \quad \frac{\mathrm{d} s}{\mathrm{~d} y}=\frac{k z \theta^{\prime \prime}}{\psi^{\prime \prime}} \frac{\mathrm{d} z}{\mathrm{~d} y}>0 .
$$

I have substituted $\varepsilon^{\prime}=\frac{1}{\theta}\left[z \theta^{\prime \prime}-(\varepsilon-1) \theta^{\prime}\right]$. All variables in the above expressions, as in the remainder of this proof, are the ones in the social optimum. Differentiating the expression for $\sigma_{e}$ in (4.13) with respect to $y$, I have:

$$
\frac{\mathrm{d} \sigma_{e}}{\mathrm{~d} y}=\left(\frac{2 \mathrm{~d} z}{\mathrm{~d} y}\right) \frac{\sigma_{e}^{2}}{x}\left[\frac{(\varepsilon-1) k z}{s+s_{0}} \frac{\theta^{\prime \prime}}{\psi^{\prime \prime}}+\frac{\varepsilon-1}{z}-\varepsilon^{\prime}\right] .
$$

A sufficient condition for $\frac{\mathrm{d} \sigma_{e}}{\mathrm{~d} y}>0$ is $\varepsilon^{\prime} \leq \frac{\varepsilon-1}{z}$. QED 


\section{References}

[1] Albrecht, J., Gautier, P. and S. Vroman, 2006, "Equilibrium Directed Search with Multiple Applications," Review of Economic Studies 73, 869-891.

[2] Baye, M.R., Morgan, J. and P. Scholten, 2004, "Price Dispersion in the Small and in the Large: Evidence from an Internet Price Comparison Site," Journal of Industrial Economics 52, 463-496.

[3] Bethune, Z., Choi, M. and R. Wright, 2018, "Frictional Goods Markets: Theory and Applications," manuscript, University of Virginia.

[4] Burdett, K. and K.L. Judd, 1983, "Equilibrium Price Dispersion," Econometrica 51, 955-969.

[5] Burdett, K. and D. Mortensen, 1998, "Wage Differentials, Employer Size, and Unemployment," International Economic Review 39, 257-273.

[6] Burdett, K. Shi, S. and R. Wright, 2001, "Pricing and Matching with Frictions," Journal of Political Economy 109, 1060-1085.

[7] Dagum, C., 1975, "A Model of Income Distribution and the Conditions of Existence of Moments of Finite Order," Bulletin of the International Statistical Institute 46, 199-205.

[8] Delacroix, A. and S. Shi, 2013, "Pricing and Signaling with Frictions," Journal of Economic Theory 148, 1301-1332.

[9] Diamond, P.A., 1971, "A Model of Price Adjustment," Journal of Economic Theory 3, 156-168.

[10] Diamond, P.A., 1982, "Aggregate Demand Management in Search Equilibrium," Journal of Political Economy 90, 881-894.

[11] Ellison, G. and S. Ellison, 2005, "Lessons about Markets from the Internet," Journal of Economic Perspectives 19 (2), 139-158.

[12] Galenianos, M. and P. Kircher, 2009, "Directed Search with Multiple Job Applications," Journal of Economic Theory 144, 445-471.

[13] Godoy, A. and E. Moen, 2013, "Mixed Search," manuscript, University of Oslo.

[14] Gourio, F. and L. Rudanko, 2014, "Customer Capital," Review of Economic Studies $81,1102-1136$.

[15] Guerrieri, G., Shimer, R. and R. Wright, 2010, "Adverse Selection in Competitive Search Equilibrium," Econometrica 78, 1823-1862.

[16] Haight, Frank A., 1967, Handbook of the Poisson Distribution. New York: John Wiley \& Sons.

[17] Julien, B., Kennes, J. and I. King, 2000, "Bidding for Labor," Review of Economic Dynamics 3, 619-649. 
[18] Kennes, J., le Maire, D. and S. Roelsgaard, 2018, "Equivalence of Canonical Matching Models," manuscript, Aarhus University.

[19] Kim, K. and P. Kircher, 2015, "Efficient Competition through Cheap Talk: The Case of Competing Auctions," Econometrica 83, 1849-1875.

[20] Lester, B., 2011, "Information and Prices with Capacity Constraints," American Economic Review 101, 1591-1600.

[21] Lester, B., Visschers, L. and R. Wolthoff, 2017, "Competing with Asking Prices," Theoretical Economics 12, 731-770.

[22] Menzio, G., 2007, "A Theory of Partially Directed Search," Journal of Political Economy $115,748-769$.

[23] Menzio, G. and S. Shi, 2010, "Block Recursive Equilibria for Stochastic Models of Search on the Job," Journal of Economic Theory 145, 1453-1494.

[24] Moen, E.R., 1997, "Competitive search equilibrium," Journal of Political Economy 105, 385-411.

[25] Montgomery, J.D., 1991, "Equilibrium Wage Dispersion and Interindustry Wage Differentials," Quarterly Journal of Economics 106, 163-179.

[26] Peters, M., 1991, "Ex Ante Price Offers in Matching Games: Non-Steady State," Econometrica 59, 1425-1454.

[27] Salop, S. and J. Stiglitz, 1977, "Bargains and Ripoffs: A Model of Monopolistically Competitive Price Dispersion," Review of Economic Studies 44, 493-510.

[28] Shi, S., 2001, "Frictional Assignment, I: Efficiency," Journal of Economic Theory 98, 232-260.

[29] Shi, S., 2009, "Directed Search for Equilibrium Wage-Tenure Contracts," Econometrica $77,561-584$.

[30] Shi, S., 2016, "Customer Relationship and Sales," Journal of Economic Theory 166, 483-516.

[31] Shi, S., 2018, "Sequentially Mixed Search Equilibrium with Many-to-Many Meetings," manuscript, Pennsylvania State University.

[32] Shi, S. and A. Delacroix, 2018, "Should Buyers or Sellers Organize Trade in a Frictional Market?", Quarterly Journal of Economics 133, 2171-2214.

[33] Shilony, Y., 1977, "Mixed Pricing in Oligopoly," Journal of Economic Theory 14, 373-388.

[34] Stacey, D., 2015, "Posted Prices, Search and Bargaining," manuscript, Ryerson University.

[35] Varian, H., 1980, “A Model of Sales," American Economic Review 70, 651-659. 


\section{For Online Publication \\ Supplementary Appendix for \\ "Sequentially Mixed Search and Equilibrium Price Dispersion"}

Shouyong Shi

\section{Proofs of Corollary 3.1, Proposition 3.3 and Lemma 4.1}

\section{Proof of Corollary 3.1:}

After substituting $e^{-x F}=\frac{\pi(x)}{p}$ and $\pi(x)=\frac{k}{\lambda(x)}$, it is straightforward to show from (2.6) and (2.5) that $\frac{\mathrm{d} F(p \mid x)}{\mathrm{d} x}<0$ and $\frac{\mathrm{d} F(p \mid x)}{\mathrm{d} x}<0$ for any given $p$. That is, an increase in $x$ increases prices in the first-order stochastic dominance in $F$ and $G$. Similarly, using (2.8) and (3.1), it can be verified that an increase in $x$ increases $\left(p_{L}, p_{H}\right), \Delta p$ and $\left(c v_{F}, c v_{G}\right)$.

To prove $p_{H}<y-b$, suppose $p_{H} \geq y-b$, to the contrary. If stayers choose to enter the submarket with such $p_{H}$, then $\Pi\left(p_{H}, x, F\left(p_{H}\right)\right)=\pi$, which can be rewritten as $p_{H} e^{-x}=\frac{k \theta(x)}{x}$. Thus,

$$
\theta^{\prime}(x)>\frac{\theta(x)}{x}=\frac{p_{H}}{k} e^{-x} \geq \frac{1}{k}(y-b) e^{-x} .
$$

Comparing this condition with (2.11) yields $x>x^{*}$. The submarket $(F, x)$ is not optimal for visitors to enter. This proves $p_{H}<y-b$. QED

\section{Proof of Proposition 3.3:}

Taking $\ln$ on (3.2) and differentiating yields:

$$
\left(\frac{\nu \theta^{\prime \prime}}{\theta^{\prime}}+x\right) \frac{\mathrm{d} x}{x}=\frac{\mathrm{d} y}{y-b}+\left(\frac{\nu \theta^{\prime \prime}}{\theta^{\prime}}+1\right) \frac{\mathrm{d} A}{A},
$$

where the asterisk on $x$ is suppressed and the argument of $\theta$ is $\nu=x / A$. Since $\theta^{\prime}>0$ and $\theta^{\prime \prime}>0$, then $\frac{\mathrm{d} x}{\mathrm{~d} y}>0$ and $\frac{\mathrm{d} x}{\mathrm{~d} A}>0$. Differentiating $\theta(\nu)$ and substituting $(\mathrm{d} x)$ yields:

$$
\left(\frac{\nu \theta^{\prime \prime}}{\theta^{\prime}}+x\right) \frac{\mathrm{d} \theta}{\nu \theta^{\prime}}=\frac{\mathrm{d} y}{y-b}+(1-x) \frac{\mathrm{d} A}{A} .
$$

Clearly, $\frac{\mathrm{d} \theta}{\mathrm{d} y}>0$ and $\frac{\mathrm{d} \theta}{\mathrm{d} A}>0$. Substituting $\pi$ from (2.9) into (2.8) and differentiating, I get:

$$
\frac{x}{k \theta}\left(\frac{\nu \theta^{\prime \prime}}{\theta^{\prime}}+x\right) \mathrm{d} p_{L}=\frac{\varepsilon-1}{y-b} \mathrm{~d} y-\left(\frac{\theta}{\theta^{\prime}} \varepsilon^{\prime}+x \varepsilon\right) \frac{\mathrm{d} A}{A}
$$




$$
\begin{aligned}
\frac{x e^{-x}}{k \theta}\left(\frac{\nu \theta^{\prime \prime}}{\theta^{\prime}}+x\right) \mathrm{d} p_{H} & =\frac{\varepsilon-1+x}{y-b} \mathrm{~d} y-\frac{\theta \varepsilon^{\prime}}{\theta^{\prime}}(1-x) \frac{\mathrm{d} A}{A} \\
\frac{x e^{-x}}{k \theta}\left(\frac{\nu \theta^{\prime \prime}}{\theta^{\prime}}+x\right) \mathrm{d} \Delta p= & \frac{\left(1-e^{-x}\right)(\varepsilon-1)+x}{y-b} \mathrm{~d} y \\
& +\left[\left(x-1+e^{-x}\right) \frac{\theta}{\theta^{\prime}} \varepsilon^{\prime}+x \varepsilon e^{-x}\right] \frac{\mathrm{d} A}{A}
\end{aligned}
$$

I have used $\varepsilon^{\prime}(\nu)=\frac{1}{\theta}\left[\nu \theta^{\prime \prime}-(\varepsilon-1) \theta^{\prime}\right]$, where the argument of $\theta$ and $\varepsilon$ is $\nu=x / A$. Because $\varepsilon>1$, the above equations show that $\frac{\mathrm{d} p_{L}}{\mathrm{~d} y}>0, \frac{\mathrm{d} p_{H}}{\mathrm{~d} y}>0$ and $\frac{\mathrm{d} \Delta p}{\mathrm{~d} y}>0$. The effects of $A$ on $\left(p_{L}, p_{H}, \Delta p\right)$ depend on $\varepsilon^{\prime}$. Note that $x-1+e^{-x}>0$ for all $x>0$. If $\varepsilon^{\prime} \geq 0$, then $\frac{\mathrm{d} p_{L}}{\mathrm{~d} A}<0$, $\frac{\mathrm{d} p_{H}}{\mathrm{~d} A} \leq 0$, and $\frac{\mathrm{d} \Delta p}{\mathrm{~d} A}>0$, where the equality on $\frac{\mathrm{d} p_{H}}{\mathrm{~d} A}$ holds if and only if $\varepsilon^{\prime}=0$.

The cumulative distribution function of posted prices is $F(p \mid x)$ given by (2.6) and the cumulative distribution function of transaction prices is $G(p \mid x)$ given by (2.5). For any given $p$, differentiating these functions with respect to $(y, A)$ yields:

$$
\begin{aligned}
& x\left(\frac{\nu \theta^{\prime \prime}}{\theta^{\prime}}+x\right) \mathrm{d} F=-\frac{\varepsilon-1+x F}{y-b} \mathrm{~d} y+\left[(1-x F) \frac{\theta}{\theta^{\prime}} \varepsilon^{\prime}+x \varepsilon(1-F)\right] \frac{\mathrm{d} A}{A} \\
& x\left(\frac{\nu \theta^{\prime \prime}}{\theta^{\prime}}+x\right) \frac{\mathrm{d} G}{G}=-\left(\frac{\varepsilon-1}{e^{x F}-1}+\frac{x}{e^{x}-1}\right) \frac{\mathrm{d} y}{y-b} \\
&+\left[\frac{\theta \varepsilon^{\prime}}{\theta^{\prime}}\left(\frac{1}{e^{x F}-1}-\frac{x}{e^{x}-1}\right)+x \varepsilon\left(\frac{1}{e^{x F}-1}-\frac{1}{e^{x}-1}\right)\right] \frac{\mathrm{d} A}{A} .
\end{aligned}
$$

Because $\varepsilon>1$, the coefficients of $(\mathrm{d} y)$ in both equations are strictly negative. Thus, an increase in $y$ increases both posted and transaction prices in the first-order stochastic dominance. If $\varepsilon^{\prime} \geq 0$, the coefficients of $(\mathrm{d} A)$ in (C.5) and (C.5) are strictly positive for all interior $p$. Thus, an increase in $A$ reduces both posted and transaction prices.

The coefficients of variation given in (3.1) are only functions of $x$. Since $c v_{F}^{\prime}(x)>0$ and $c v_{G}^{\prime}(x)>0$ (see Corollary 3.1), the above results $\frac{\mathrm{d} x}{\mathrm{~d} y}>0$ and $\frac{\mathrm{d} x}{\mathrm{~d} A}>0$ imply $\frac{\mathrm{d}}{\mathrm{d} y} c v_{i}(x)>0$ and $\frac{\mathrm{d}}{\mathrm{d} A} c v_{i}(x)>0$ for $i=F, G$. QED

\section{Proof of Lemma 4.1:}

Using L'Hopital's rule, one can verify the properties in (i) of Lemma 4.1. To verify $L(t)>0$ in (ii) of the Lemma, note that $1>e^{-t}$ if and only if $t>0$. Thus, $L(t)>0$ for all $t \neq 0$. In addition, $L(0)=1>0$, as proven in part (i). To prove $L^{\prime}(t)<0$ for all $t$, it suffices to prove $L^{\prime}(t)<0$ for all $t \neq 0$, since $L^{\prime}(0)=-\frac{1}{2}<0$ by (i). Compute

$$
L^{\prime}(t)=\frac{1}{t^{2}}\left[(t+1) e^{-t}-1\right] .
$$


Examine the expression in [.] on the right-hand side for $t \neq 0$. The derivative of the expression with respect to $t$ is equal to $-t e^{-t}$, which is positive if and only if $t<0$. Thus, the expression is maximized as $t \rightarrow 0$. Because the expression approaches 0 as $t \rightarrow 0$, the expression is negative for all $t \neq 0$. Thus, $L^{\prime}(t)<0$ for all $t \neq 0$. Similarly, compute:

$$
L^{\prime \prime}(t)=\frac{1}{t^{3}}\left[2-\left(t^{2}+2 t+2\right) e^{-t}\right] .
$$

The expression in [.] on the right-hand side is increasing in $t$ and, at $t=0$, it is equal to 0 . Thus, the expression is positive if and only if $t>0$. Since $t^{3}>0$ if and only if $t>0$, then $L^{\prime \prime}(t)>0$ for all $t$. QED 